IIIIIIII BANK OF CANADA

BANQUE DU CANADA

Staff Working Paper/Document de travail du personnel - 2020-50

Last updated: November 26, 2020

\title{
A Macroeconomic Model of an Epidemic with Silent Transmission and Endogenous Self-isolation
}

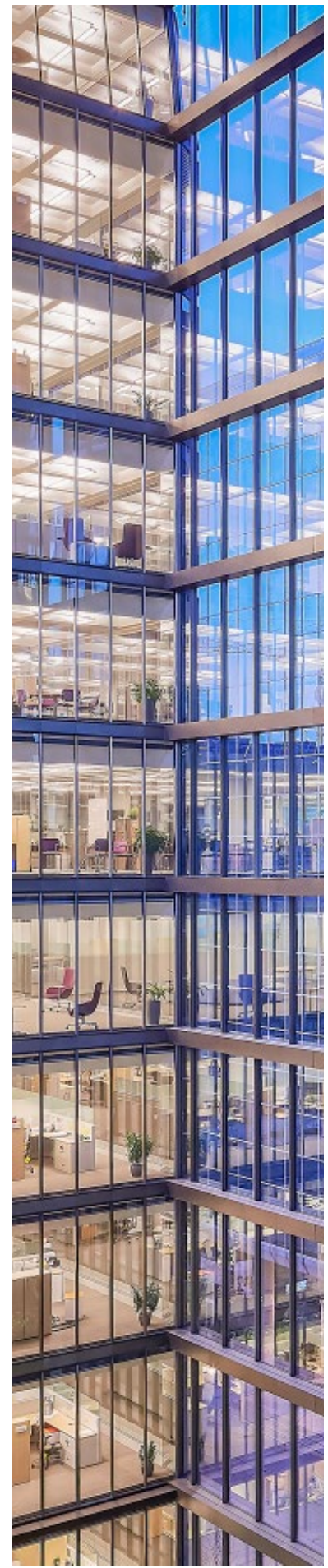

Funds Management and Banking Department Bank of Canada, Ottawa, Ontario, Canada K1A 0G9

adiez@bankofcanada.ca

Bank of Canada staff working papers provide a forum for staff to publish work-in-progress research independently from the Bank's Governing Council. This research may support or challenge prevailing policy orthodoxy. Therefore, the views expressed in this paper are solely those of the authors and may differ from official Bank of Canada views. No responsibility for them should be attributed to the Bank. 


\section{Acknowledgements}

I would like to thank James Chapman, Jonathan Chiu, Maryam Haghighi, Maren Hansen, Oleksiy

Kryvtsov and seminar participants at the Bank of Canada for their suggestions. Any (remaining) errors are my own. The views expressed in this paper are those of the author and do not necessarily reflect those of the Bank of Canada. 


\section{Abstract}

We study the interaction between epidemics and economic decisions in a model where (1) agents allocate their time to market and home production and social and home leisure, (2) these activities differ in their degree of contagiousness, (3) some infected individuals are indistinguishable from susceptible individuals, and (4) agents are not necessarily rational. For baseline parameter values for the COVID-19 pandemic, we find that agents partially selfisolate by allocating more time to home activities and that the effective reproduction number of the disease stabilises at one. Detection and isolation of infected individuals severely mitigate the recession caused by the pandemic.

Topics: Coronavirus disease (COVID-19); Economic models

JEL codes: E1, HO, 11 


\section{Introduction}

As the world continues to grapple with the global spread of the Coronavirus disease (COVID-19), there is increasing evidence that most mild and asymptomatic COVID-19 cases tend to go undiagnosed or unreported. For example, Li et al. (2020) estimate that 86 per cent of all COVID-19 infections in China were undocumented before the January 23, 2020, travel restrictions imposed to curtail the spread of the disease. Ing, Cocks and Green (2020) found that 81 per cent of COVID-19 infected individuals travelling on an expedition cruise ship to Antarctica were asymptomatic. Poletti et al. (2020), using data on contacts of COVID-19 index cases detected in Lombardy (Italy), found that nearly 75 per cent of all infected individuals aged less than 60 years did not present recognizable symptoms. Moghadas et al. (2020) found that the majority of new COVID-19 infections may be attributable to silent transmissions from a combination of presymptomatic and asymptomatic cases. In this paper, we study the interaction between epidemics and economic decisions in a model where there is silent transmission of the disease. Our results, based on a calibrated version of the model, suggest that supplementing symptombased isolation with detection and isolation policies targeted at asymptomatic or mildly symptomatic cases can have a strong impact on reducing the severity of the recession induced by the COVID-19 pandemic.

Our starting point is the canonical macro-epidemiology model of Eichenbaum, Rebelo and Trabandt (2020a) (hereafter, ERTa), which extends the classic susceptible-infectedrecovered (SIR) model of Kermack and McKendrick (1927). As a main difference with the SIR model, ERTa allows the probability of becoming infected with the disease to depend on people's economic decisions. Importantly, ERTa find that people's decisions to cut back on consumption and work saves lives but exacerbates the severity of the recessions caused by the epidemic. In this paper, we build on ERTa's framework to study the economic implications of an epidemic with silent transmission in which agents can choose to self-isolate. ${ }^{1}$

First, agents in our model solve a time allocation problem (see, e.g., Becker, 1965 and Gronau, 1977) in which they need to decide how much time to devote to the production of a market good, the production of a home good, social leisure and leisure at home and where their probability of becoming infected with the disease depends on the time spent

\footnotetext{
${ }^{1}$ Throughout this paper, we define isolation as the complete separation of a person infected with the disease from other individuals (e.g., by staying at home). On the other hand, we define self-isolation as the decision of a susceptible person to stay at home to minimize their probability of being infected.
} 
in each of these activities. In this way, we allow the agents' decisions regarding their social interactions (in addition to their economic decisions) to affect their probability of becoming infected since, as noted in the clinical literature, a substantial number of the overall transmissions of respiratory diseases do not occur as a result of economic activity (e.g., Ferguson et al., 2006).

Importantly, the agents in our model can fully self-isolate. That is, the probability of becoming infected is zero when they decide to stay at home (devoting time to either home production or home leisure).

Second, we follow Krueger, Uhlig and Xie (2020) (hereafter, KUX), who extend the model in ERTa to the case of several highly substitutable goods in the economy that differ in their degree of contagiousness (e.g., pizza delivery versus eating pizza in a restaurant), in assuming that home and market goods are imperfect substitutes. We differ from KUX, who assume that different sectors in the economy have the same productivity, in that we assume that the home production sector has a lower marginal productivity (see, e.g., Benhabib, Rogerson and Wright, 1991).

Third, we assume that there are two different types of infected individuals. Some of them develop a severe form of the illness, which we model as the combination of (i) a reduction in the infected individual's time available for work and leisure and (ii) a positive probability of dying as a consequence of the disease. Further, in order to focus on the effects of silent transmission, we assume that individuals with severe symptoms are isolated immediately and, therefore, do not contribute to the spread of the disease. In addition, we assume that there is a second group of infected individuals in our model that present a mild form of the illness but can transmit the disease to others.

Importantly, in the absence of a detection mechanism (i.e., testing), mildly symptomatic infected individuals cannot be distinguished from healthy agents and, thus, contribute to the silent transmission of the epidemic. Similar to Eichenbaum, Rebelo and Trabandt's (2020b) findings (hereafter, ERTb), this dichotomy in the presentation of the disease implies that there are individuals in our model who do not know their health status (e.g., whether they are infected with the disease, have recovered or remain susceptible). We assume, in line with the literature, that agents are immune to the disease once they recover (e.g., ERTa and KUX).

Rational agents. For the baseline parameter values for the COVID-19 pandemic, we find that when agents are rational (i.e., they are aware of the initial outbreak and under- 
stand the laws of motion governing the population health dynamics), they partially shield themselves from the epidemic by reallocating their time working in the market and on social activities to home activities. For example, we find that the time a rational agent spends in out-of-the-house activities is cut almost in half at the peak of the epidemic (27 per cent versus a pre-epidemic steady-state allocation of 50 per cent). Specifically, the rational agents reallocate their time such that the effective reproduction number of the disease (i.e., the total expected number of cases directly generated by one infected person) stabilizes at one (see also Gans, 2020, for a similar result). That is, these agents behave in way that balances avoiding exponential growth in the disease with their preference to spend more time outside of their homes. Consequently, the number of total infections grows at a linear pace, thus, slowing down the spread of the disease if compared with the case of a model where the agents are naïve (i.e., do not change their behaviour).

As in ERTa and KUX, we find that rational behaviour is a strong mitigator of the severity of the epidemic as measured by the number of total infections (and consequently, the number of deaths). For example, we find that, in a model with rational agents, only roughly 10 per cent of the population becomes infected by the end of the third year after the outbreak (week 150) compared with almost 80 per cent of the population being infected by week 35 in the case of a model where the agents are naïve. The number of deaths at the end of the third year after the epidemic is ten times lower than in a model where the agents are naïve: 0.016 per cent versus 0.16 per cent, respectively.

However, the agents' endogenous response exacerbates the depth and length of the recession that is caused by the epidemic, given that these agents shield themselves by reallocating their time from the production of the market good to home production. Since the home good has lower productivity, aggregate consumption falls. For example, in a model with rational agents we find that aggregate consumption falls by roughly 9 per cent from its pre-epidemic steady-state value by week 25 (bottom of the recession) versus 2.5 per cent for the case of a naïve agent. Importantly, halting the progression of the epidemic results in a protracted recession. For example, aggregate consumption is still 7 per cent lower than in the pre-epidemic steady state by week 150 after the outbreak when the agents are rational, while it is only 0.16 per cent lower when the agents are naïve (consistently with the death of 0.16 per cent of the workforce).

Isolating mildly symptomatic infected individuals. Our results indicate that, when there is silent transmission of the disease, detection and isolation of mildly symp- 
tomatic cases is a very powerful tool to mitigate the economic impact of the epidemic, thus, providing support for the testing policies advocated by Romer (2020). First, detection and isolation policies reduce the infection risk faced by susceptible agents, leading to more production and consumption of the market good. Second, detection and isolation policies reduce the size of the pool of people that do not know they have recovered (and, consequently, reduce their out-of-the-house activities when they are, in fact, already immune to the disease). ${ }^{2}$ For example, when the isolation of severely ill patients is complemented with the isolation of 40 per cent of mildly symptomatic individuals, we find that a recession is essentially avoided: aggregate consumption falls only 0.035 per cent from the pre-epidemic steady state.

As expected, the fraction of deaths by the end of the third year also falls by an order of magnitude: from 0.016 per cent in the case of no isolation to 0.0015 per cent in the case where 40 per cent of mildly symptomatic individuals are isolated. Still, since the number of deaths is already relatively small (when compared to a model with naïve agents), we find that the main outcome of detecting and isolating mildly symptomatic cases is a less severe recession.

Optimistic agents. The idea KUX put forward about individual behaviour being an endogenous mitigation mechanism for the epidemic strongly relies on all individuals fully understanding the health population dynamics, in general, and the dangers of exponentially growing epidemics in particular. To analyze the robustness of this result, we also depart from ERTa and KUX in assuming that the agents in our model might not be fully rational. Specifically, motivated by the work by Gabaix (2020), we assume that the agents in our model show a form of cognitive discounting in that they neither fully understand the post-outbreak world nor how their behaviour affects their probability of becoming infected. ${ }^{3}$ To capture this mathematically, we assume that the agents forecast the future by shrinking their expectations toward the pre-epidemic steady state of the economy where there is no spread of the disease: the higher the weight in the economy's pre-epidemic steady state, the more inattentive the agent is. This is motivated by the psychology of "anchoring and adjustment" in that people start from an initial default value that is adjusted to make the final estimate but where the adjustment is usually insufficient (see,

\footnotetext{
${ }^{2}$ We note that, given the linear production function for the market good in equation (3), this channel embodies both the consumption and work channels in the model in ERTa.

${ }^{3}$ See Halpern, Truog and Miller (2020) for a discussion of the role of cognitive biases and public health policies during the COVID-19 pandemic.
} 
i.e., Tversky and Kahneman, 1974). ${ }^{4}$ In our case, this insufficient adjustment results in agents that are optimistic: they underestimate the probability of becoming infected, the probability of developing a severe form of the illness and the mortality rate.

When agents are optimistic (but not too close to being naïve), we find a pattern in the evolution of the epidemic similar to the one documented for the case of full rationality: agents still understand the dangers of exponentially growing epidemics, and the effective reproduction rate of the disease stabilizes at one. However, optimistic agents underestimate the cost of being infected with the disease since they underestimate the likelihood of developing severe symptoms and dying. Consequently, they delay their response to the epidemic, which leads to a higher peak of infections and, in turn, to a recession that is similar in size to that in the case of the model with rational agents. For example, we find that in a model with inattentive agents and no detection and isolation policies, roughly 26 per cent of the population becomes infected by the end of the third year after the outbreak and aggregate consumption falls by 8 per cent from its pre-epidemic steady state (compared with 10 per cent of the population becoming infected by the end of the third year and aggregate consumption falling by 9 per cent when agents are rational).

Epidemic waves. Finally, we analyze a model where the agents are initially inattentive to the outbreak but they become more attentive over time. Similar to Cochrane (2020), we find that such a model can generate epidemic waves. Specifically, we assume that the agents pay more attention to the epidemic when the number of new deaths in the last quarter is sufficiently high and as time goes by (i.e., the pre-epidemic steady state is more distant). Our results indicate that inattention to its initial phase allows the epidemic to gather a lot of momentum. As the number of new deaths increases rapidly, agents become more attentive and practically self-isolate. This abrupt time reallocation causes an even deeper recession (27 per cent fall in aggregate consumption and no isolation of mildly symptomatic cases) if compared to that in the models with rational expectations (9 per cent fall) and constant inattention (8 per cent fall).

The agents' aggressive response sets the stage for a second wave in the epidemic, given that as the number of infections and new deaths fall, agents stop paying attention to the epidemic and attempt to return too quickly to their pre-epidemic steady-state allocation of time. This, in turn, causes infections and deaths to spike again and the agents to shield

\footnotetext{
${ }^{4}$ Importantly, this framework nests the case of naïve and rational agents. When the weight in the pre-epidemic steady state is one, we have naïve agents that do not change their behaviour. When the weight in the pre-epidemic steady state is zero, we return to the case of rational agents.
} 
themselves once more by increasing their time at home. Aggregate consumption does not fall as much in the second wave of the epidemic (12.5 per cent versus 27 per cent), yet this fall is still larger than the drop in consumption in the models with rational and constant inattention. Eventually, since we assume that the agents pay full attention to the epidemic by the end of the second year, the model predictions become very similar to those of the model with rational agents.

Importantly, the detection and isolation of asymptomatic and mildly symptomatic infected individuals is even more important than in the case of a model with rational agents, given that both the economic and health outcomes are substantially worse in a model where the agents are initially inattentive to the outbreak .

Literature review. Our results are complementary to those emerging from a quickly growing literature on the macroeconomic impact of the COVID-19 pandemic including, among others, Acemoglu et al. (2020); Alvarez, Argente and Lippi (2020); Bethune and Korinek (2020); Berger, Herkenhoff and Mongey (2020); Cochrane (2020); ERTa; ERTb; ERTc; Jones, Philippon and Venkateswaran (2020); Glover et al. (2020); Guerrieri, et al. (2020); Kaplan, Moll and Violante (2020), KUX and Toxvaerd (2020). Our main difference with respect to these papers is our focus on the impact of the silent spread of the COVID-19 pandemic on the economy and, consequently, on the detection and isolation of mildly symptomatic infected individuals.

This paper relates to the literature that studies the macroeconomic implications of including household production in macroeconomic models. These papers include, for example, Gronau (1977, 1986); Benhabib, Rogerson and Wright (1991); Greenwood and Hercovitz (1991) and, more recently, Aruoba, Davis and Wright (2016) and Gayle and Shephard (2019). These papers try to formalize the agents' decision to work in the market, work at home and engage in leisure. We contribute to this literature by considering two different types of leisure: social and home leisure.

On the behavioural front, this paper is also related to the literature on macroeconomic models with bounded rationality microfounded by the use of the "sparsity" approach introduced by Gabaix (2014) and applied to dynamic macroeconomics models in Gabaix (2016, 2020), and public economics in Farhi and Gabaix (2020). Similarly, our paper also relates to the literature on inattention to health consequences in which agents underperceive the future health costs of their decisions (see, e.g., Gruber and Koszegi, 2001, and Allcott, Lockwood and Taubinsky, 2019). 
Paper outline. The rest of this paper is organized as follows. We present an SIR macro model with home production and home leisure in Section 2. Section 3 presents the details of the calibration. Section 4 discusses the results of the models with naïve and rational agents, while Section 5 presents the results with behavioural agents. Section 6 concludes.

\section{An SIR model with home production and home leisure}

\subsection{The pre-infection economy}

The economy is populated by a continuum of individuals with unit mass and preferences over four objects - consumption of a market good $\left(c_{k, t}\right)$, consumption of a home-produced good $\left(c_{h, t}\right)$, social leisure time (i.e., outside of the home) $\left(l_{k, t}\right)$ and leisure time at home $\left(l_{h, t}\right)$ - described by

$$
U_{0}=E_{0} \sum_{t=0}^{\infty} \beta^{t} u\left(c_{k, t}, c_{h, t}, l_{k, t}, l_{h, t}\right),
$$

where $E_{t}$ denotes the conditional expectation given the information available up (and including) to time $t$ (measured in weeks), and $\beta$ denotes the discount factor.

Agents have $\bar{n}$ units of time to divide between leisure and labour each period (which we will later normalize to $\bar{n}=1$ for the case of healthy individuals and assume that $\bar{n}<1$ for infected individuals with severe symptoms). Specifically, agents decide how much time to allocate to working in the market sector $\left(n_{k, t}\right)$, home production $\left(n_{h, t}\right)$ and to leisure in its two forms (social leisure, $l_{k, t}$, and at-home leisure, $l_{h, t}$ ):

$$
\bar{n}=n_{k, t}+n_{h, t}+l_{k, t}+l_{h, t}
$$

Time spent working in the market sector is used to produce market goods according to a linear technology:

$$
c_{k, t}=A_{k} n_{k, t}
$$

while time spent working in the home is used to produce home goods in a similar fashion

$$
c_{h, t}=A_{h} n_{h, t}
$$

Consistent with Benhabib, Rogerson and Wright (1991), we assume that $A_{h}<A_{k}$. That is, the productivity in the home sector is lower than that in the market sector..$^{5,6}$

\footnotetext{
${ }^{5}$ Similar to ERTa, we assume the existence of a competitive representative firm of unit measure that produces market goods using the time worked in the market sector and that pays the agent a real salary $w_{t}$. In equilibrium, $w_{t}=A_{k}$ and consequently, equation (3) can be viewed as the agent's budget constraint.

${ }^{6}$ In a more general setup, we could allow for producing the market good with two types of labour that
} 
We specify the momentary utility function as

$$
u\left(c_{t}, l_{t}\right)=\theta_{0}+\ln \left(c_{t}\right)+\theta_{l} \ln \left(l_{t}\right)
$$

Similar to KUX, we assume that the consumption is a bundle across the home and market goods:

$$
c_{t}=\left(\omega_{h}^{c} c_{h, t}^{\frac{\eta-1}{\eta}}+c_{k, t}^{\frac{\eta-1}{\eta}}\right)^{\frac{\eta}{\eta-1}},
$$

where $\eta \geq 0$ is the elasticity of substitution between market and home good consumption. In contrast to KUX, we also assume that the representative agent enjoys a bundle of home and social leisure:

$$
l_{t}=\left(\omega_{h}^{l} l_{h, t}^{\frac{\sigma-1}{\sigma}}+l_{k, t}^{\frac{\sigma-1}{\sigma}}\right)^{\frac{\sigma}{\sigma-1}}
$$

where $\sigma \geq 0$ is the elasticity of substitution between social and home leisure. Finally, $\theta_{0}$ is a constant used to calibrate the value of a human life for the representative agent, as in Hall and Jones (2007) and ERTb. ${ }^{7}$

We show in the appendix that the solution to the maximization problem of the representative agent with preferences given by (1) and (5) subject to the constraints (2), (3) and (4) is given by:

$$
\begin{gathered}
n_{k, t}=\frac{1}{\left[\left(\frac{A_{h}}{A_{k}}\right)^{\eta-1}\left(\omega_{h}^{c}\right)^{\eta}+1\right]} \frac{\bar{n}}{\left(1+\theta_{l}\right)}, \\
n_{h, t}=\frac{\left(\frac{A_{h}}{A_{k}}\right)^{\eta-1}\left(\omega_{h}^{c}\right)^{\eta}}{\left[\left(\frac{A_{h}}{A_{k}}\right)^{\eta-1}\left(\omega_{h}^{c}\right)^{\eta}+1\right]} \frac{\bar{n}}{\left(1+\theta_{l}\right)}, \\
l_{k, t}=\frac{1}{\left[\left(\omega_{h}^{l}\right)^{\sigma}+1\right]} \frac{\theta_{l} \bar{n}}{\left(1+\theta_{l}\right)}, \\
l_{h, t}=\frac{\left(\omega_{h}^{l}\right)^{\sigma}}{\left[\left(\omega_{h}^{l}\right)^{\sigma}+1\right]} \frac{\theta_{l} \bar{n}}{\left(1+\theta_{l}\right)} .
\end{gathered}
$$

Note that $n_{k, t}+n_{h, t}=\bar{n} /\left(1+\theta_{l}\right)$ captures the total amount of time devoted to work, and $l_{k, t}+l_{h, t}=\theta_{l} \bar{n} /\left(1+\theta_{l}\right)$ is the amount of time devoted to leisure.

\subsection{The epidemic: objective reality}

Population groups. In our model, the population is divided into seven groups:

differ in their degree of contagiousness (e.g., working in the office versus tele-commuting). Similarly, we could allow for the market good to be an additional input in the production of the home good (e.g., flour to bake bread at home). We leave such extensions for future work.

${ }^{7}$ We follow Hall and Jones (2007) and define the value of a human life in units of output for the representative agent as $L\left(c_{t}\right) \equiv u\left(c_{t}\right) / u^{\prime}\left(c_{t}\right)$. 
1. The susceptible people of mass $S_{t}$ who have not yet been exposed to the disease.

2. The infected people of mass $I_{t}$ who have contracted the disease and present severe (identifiable) symptoms. We model the severe form of the disease as a decrease in the amount of time that a representative infected individual can devote to work and leisure (i.e., from $\bar{n}=1$ to $\bar{n}^{i}<1$ ) and a positive probability of dying. In order to focus on the effects of a silent transmission of the disease, we assume that infected individuals with severe symptoms are detected and isolated immediately $\left(n_{k, t}^{i}=l_{k, t}^{i}=0\right)$.

3. People experiencing a mild form of the illness have mass $A_{t}$. These people have contracted the disease (and therefore are contagious) but show only mild or no symptoms. Consequently, we assume that the mortality rate for these individuals is zero and that they have the same amount of time available to them for work and leisure as does the susceptible group $(\bar{n}=1)$. Further, in the absence of any detection mechanism, these individuals do not know whether they have been infected (i.e., they are indistinguishable from susceptible people).

4. On the other hand, infected individuals with mild symptoms are forced to stay at home if detected $\left(n_{k, t}^{q}=l_{k, t}^{q}=0\right)$. We refer to this group as the isolated infected people of mass $Q_{t}$.

5. The recovered people, who know that they survived the disease and acquired immunity, have mass $R_{t}$. This group of people know they have recovered because they either had a severe form of the disease or mild symptoms and were detected (and therefore isolated). ${ }^{8}$

6. In contrast, there is a group of undetected recovered individuals with mass $Z_{t}$ that, since they experienced a mild illness and their disease went undetected, do not know they survived the disease and recovered.

7. Finally, there is a mass $D_{t}$ of deceased individuals who did not survive the disease.

Each type of individual will allocate their time in a different way, depending on their probability of becoming infected, the severity of their symptoms and whether they are

\footnotetext{
${ }^{8}$ See Bethune and Korinek (2020) and ERTb's macro-epidemiology models where there is the possibility of reinfection.
} 
isolated or have recovered. Consequently, let $\left(n_{k, t}^{j}, n_{h, t}^{j}, l_{k, t}^{j}, l_{h, t}^{j}\right)^{\prime}$ be the allocation of time of a type- $j$ agent for $j=\{s, i, a, q, r, z, d\} .^{9}$

Probability of infection. In the spirit of KUX, we assume that different activities differ in their contagiousness. Let $\tau_{t}$ be the objective probability that a susceptible person becomes infected, which we assume depends on their own allocation of time across market, social and home activities:

$$
\tau_{t}=\pi_{n} n_{k, t}^{s}\left(A_{t} n_{k, t}^{a}\right)+\pi_{l} l_{k, t}^{s}\left(A_{t} l_{k, t}^{a}\right)+\pi_{h, t}\left(n_{h, t}^{s}+l_{h, t}^{s}\right)\left[A_{t}\left(n_{h, t}^{a}+l_{h, t}^{a}\right)\right]
$$

That is, susceptible people can become infected in three different ways. First, susceptible people can meet infected people while working in the market sector. The probability of being exposed to the disease while in the workplace is given by $\pi_{n} n_{k, t}^{s}\left(A_{t} n_{k, t}^{a}\right)$. This probability increases with $n_{k, t}^{s}$, the amount of time that a susceptible person works in the market, and with $A_{t} n_{k, t}^{a}$, the aggregate amount of time that infected people with mild symptoms spend working in the market. The parameter $\pi_{n}$ controls the probability of becoming infected as a result of workplace interaction.

Second, susceptible people can meet infected people in the context of social leisure activities. This is captured by the term $\pi_{l} l_{k, t}^{s}\left(A_{t} l_{k, t}^{a}\right)$ in equation (12). Again, the probability of becoming infected in this way increases with $l_{k, t}^{s}$, the amount of time that a susceptible person devotes to social leisure, and $A_{t} n_{k, t}^{a}$, the total amount of time that infected people with mild or no symptoms devote to these activities. The parameter $\pi_{l}$ controls the probability of becoming infected as a result of these social interactions.

Third, we assume that susceptible people can also meet infected people at home (i.e., other members of the household). This transmission within the household is captured by the term $\pi_{h, t}\left(n_{h, t}^{s}+l_{h, t}^{s}\right)\left[A_{t}\left(n_{h, t}^{a}+l_{h, t}^{a}\right)\right]$ in equation (12). The probability of transmission in the household increases with the amount of time a susceptible person spends at home, $n_{h, t}^{s}+l_{h, t}^{s}$, and the aggregate amount of time infected people with mild or no symptoms spend at home, $A_{t}\left(n_{h, t}^{a}+l_{h, t}^{a}\right)$. We further assume that transmission in the household can only happen if susceptible individuals also spend time outside of the house. For this reason, we assume that $\pi_{h, t}=\pi_{h}\left(n_{k, t}^{s}+l_{k, t}^{s}\right)$. We view this as a reduced-form approach to model that the more time susceptible individuals spend in activities around the house, the lower the probability of becoming infected through this channel. ${ }^{10}$

\footnotetext{
${ }^{9}$ We trivially assume that the time allocation of a deceased individual is $n_{k, t}^{d}=n_{h, t}^{d}=l_{k, t}^{d}=l_{h, t}^{d}=0$.

${ }^{10} \mathrm{As}$ it is traditional in the epidemiology literature and similar to the macro model in ERT, we assume a quadratic specification for the "matching technology" in that the new infections depend on the product
} 
Importantly, in our model, a susceptible individual can drive their probability of infection to zero by fully self-isolating $\left(n_{k, t}^{s}=l_{k, t}^{s}=0\right)$. However, susceptible individuals enjoy both the market good and social leisure and, for this reason, we advance that, in equilibrium, they are are not willing to fully self-isolate.

Finally, we note that the three different ways in which an agent can be infected loosely match the three major modes through which respiratory diseases are transmitted, as discussed in Ferguson et al. (2006): (i) transmissions that occur in schools and workplaces, (ii) in the general community and (iii) in the household.

Population health dynamics. Figure 1 presents the natural history of the disease. It summarizes the dynamics of the different groups, which we now describe. ${ }^{11}$

The number of susceptible people at time $t+1$ is equal to the number of susceptible people at time $t\left(S_{t}\right)$ minus the number of people infected at time $t\left(T_{t}\right)$ :

$$
S_{t+1}=S_{t}-T_{t}
$$

where $T_{t}=\tau_{t} S_{t}$.

Further, we assume that only a fraction $\pi_{i}$ of newly infected individuals develop severe symptoms. Therefore, the number of infected people with severe symptoms at time $t+1$ is equal to the number of infected people with severe symptoms at time $t\left(I_{t}\right)$ plus the number of newly infected individuals with severe symptoms $\left(\pi_{i} T_{t}\right)$ minus the number of infected people with severe symptoms that either recovered $\left(\pi_{r}^{i} I_{t}\right)$ or died $\left(\pi_{d}^{i} I_{t}\right)$ :

$$
I_{t+1}=I_{t}+\pi_{i} T_{t}-\left(\pi_{r}^{i}+\pi_{d}^{i}\right) I_{t}
$$

where $\pi_{r}^{i}$ is the probability that an infected individual with severe symptoms recovers from the infection during period $t$, and $\pi_{d}^{i}$ is the mortality rate conditional on developing severe symptoms. ${ }^{12}$

Further, we assume that a fraction $\alpha$ of the $\left(1-\pi_{i}\right)$ infected individuals experiencing a mild form of the illness are detected and, consequently, isolated. ${ }^{13}$ Thus, the number

of the time susceptible and infected populations allocate for an activity. However, this assumption could be relaxed as in Acemoglu et al. (2020). We leave this for further research.

${ }^{11}$ Several extensions to our framework are possible. For example, we could allow for the possibility of presymptomatic transmission by allowing for some individuals with mild or no symptoms to develop a serious form of the illness. Similarly, we could allow for some undetected recovered individuals to find out they have recovered (i.e., through serology testing for antibodies). We leave these extensions for further research.

${ }^{12}$ For simplicity, we abstract from a time-varying mortality rate as in ERTa and ERTb.

${ }^{13}$ Detection of the illness is closely related to testing. However, as noted by ERTb and Berger, Herkenhoff and Mongey (2020), modelling the macroeconomic effects of testing is challenging due to imperfect 
of isolated infected individuals at time $t+1$ is equal the number of isolated people at time $t\left(Q_{t}\right)$ plus the number of newly infected individuals showing mild (or no) symptoms that are detected $\left(\left(1-\pi_{i}\right) \alpha T_{t}\right)$ minus the number of isolated infected individuals that recovered $\left(\pi_{r}^{a} Q_{t}\right)$ :

$$
Q_{t+1}=Q_{t}+\left(1-\pi_{i}\right) \alpha T_{t}-\pi_{r}^{a} Q_{t}
$$

where $\pi_{r}^{a}$ is the probability that an asymptomatic individual recovers from the infection during period $t$. As noted earlier in the description of the different groups, we assume that the mortality rate conditional on being asymptomatic is zero. Further, we assume that $\pi_{r}^{a}=\pi_{r}^{i}+\pi_{d}^{i}$

The number of undetected infected individuals with a mild form of the illness at time $t+1$ is equal the number of undetected infected people with mild symptoms at time $t\left(A_{t}\right)$ plus the number of new individuals with mild symptoms that are not detected $\left(\left(1-\pi_{i}\right)(1-\alpha) T_{t}\right)$ minus the number of undetected individuals that recovered $\left(\pi_{r}^{a} A_{t}\right)$ :

$$
A_{t+1}=A_{t}+\left(1-\pi_{i}\right) \alpha T_{t}-\pi_{r}^{a} A_{t}
$$

Similar to ERTa, we have that the timing convention that is implicit in equations (14), (15) and (16) is as follows: First, social and economic interactions occur at the beginning of time $t$ (infected and susceptible people meet). Second, changes in health status unrelated to these interactions (recovery or death) occur. Finally, at the end of period $t$, the consequences of these interactions materialize: $T_{t}$ susceptible people become infected (out of which some become severely ill, some become quarantined, and some infected people with mild symptoms remain undetected).

The number of recovered people at time $t+1$ is the number of recovered people at time $t\left(R_{t}\right)$ plus the number of severely ill and isolated individuals who just recovered $\left(\pi_{r}^{i} I_{t}\right.$ and $\pi_{r}^{a} Q_{t}$, respectively):

$$
R_{t+1}=R_{t}+\pi_{r}^{i} I_{t}+\pi_{r}^{a} Q_{t}
$$

The number of undetected recovered individuals at time $t+1$ is the number of undetected recovered people at time $t\left(Z_{t}\right)$ plus the number of infected individuals with a mild form of the illness who recover during this period:

$$
Z_{t+1}=Z_{t}+\pi_{r}^{a} A_{t}
$$

testing (i.e., the possibility of false positives and negatives) and the heterogeneity induced by the fact that individuals who are tested at different moments in time will form different subjective probabilities about their health statuses. For this reason, and in the spirit of Li et al. (2020), we model the detection of the illness rather than the testing itself. 
Finally, the number of deceased people at time $t+1$ is the number of deceased individuals at time $t\left(D_{t}\right)$ plus the number of infected people with severe symptoms who just died:

$$
D_{t+1}=D_{t}+\pi_{d}^{i} I_{t}
$$

Initial outbreak. As in ERTa, we complete the population health dynamics by assuming that a fraction $\varepsilon$ (sufficiently small) is infected at time zero (e.g., through zoonotic exposure). Consequently, we have that $I_{0}=\pi_{i} \varepsilon, Q_{0}=\left(1-\pi_{i}\right) \alpha \varepsilon, A_{0}=\left(1-\pi_{i}\right)(1-\alpha) \varepsilon$, $S_{0}=1-\varepsilon$ and $R_{0}=Z_{0}=D_{0}=0$.

\subsection{Choices of recovered and detected, isolated and severely ill individuals}

In this section, we describe the optimal time allocations of recovered and detected individuals, isolated infected individuals, and infected individuals with severe symptoms. Such individuals face no uncertainty over their health status and no intertemporal trade-offs between consumption and the probability of being infected. Consequently, these agents solve for their optimal allocations of time, period by period.

We leave the description and discussion of the optimization problem for the other three types of individuals for the next subsection, given that these agents do not know their health status and, consequently, need to form beliefs over whether they are susceptible, infected with mild symptoms or have recovered (yet undetected).

Recovered individuals. Let the lifetime utility of a recovered individual, $U_{t}^{r}$, be

$$
U_{t}^{r}=u\left(c_{t}^{r}, l_{t}^{r}\right)+\beta U_{t+1}^{r}
$$

We note that the optimization problem of the recovered individuals coincides with 
that of the representative agent in the pre-infection economy. Consequently

$$
\begin{gathered}
n_{k, t}^{r}=\frac{1}{\left(1+\theta_{l}\right)} \frac{1}{\left[\left(\frac{A_{h}}{A_{k}}\right)^{\eta-1}\left(\omega_{h}^{c}\right)^{\eta}+1\right]} \bar{n}, \\
n_{h, t}^{r}=\frac{1}{\left(1+\theta_{l}\right)} \frac{\left(\frac{A_{h}}{A_{k}}\right)^{\eta-1}\left(\omega_{h}^{c}\right)^{\eta}}{\left[\left(\frac{A_{h}}{A_{k}}\right)^{\eta-1}\left(\omega_{h}^{c}\right)^{\eta}+1\right]} \bar{n} \\
l_{k, t}^{r}=\frac{\theta_{l}}{\left(1+\theta_{l}\right)} \frac{1}{\left[\left(\omega_{h}^{l}\right)^{\sigma}+1\right]} \bar{n} \\
l_{h, t}^{r}=\frac{\theta_{l}}{\left(1+\theta_{l}\right)} \frac{\left(\omega_{h}^{l}\right)^{\sigma}}{\left[\left(\omega_{h}^{l}\right)^{\sigma}+1\right]} \bar{n} .
\end{gathered}
$$

Once infected individuals recover, they become immune and return to their pre-epidemic allocation of time.

Isolated infected individuals. The lifetime utility of an isolated (mildly symptomatic) infected individual, $U_{t}^{q}$, is given by

$$
U_{t}^{q}=u\left(c_{t}^{q}, l_{t}^{q}\right)+\beta\left[\pi_{r}^{a} U_{t+1}^{r}+\left(1-\pi_{r}^{a}\right) U_{t+1}^{q}\right] .
$$

Once again, given the structure of the optimization problem, we have that agents solve for their optimal allocations of time, period by period. An isolated infected individual will maximize their momentary utility function subject to the constraints in (2), (3) and (4) and the isolation restrictions $n_{k, t}^{q}=l_{k, t}^{q}=0$, given that infected individuals are forced to stay at home to limit the spread of the disease.

Consequently, we have that the optimal allocation of time of an isolated infected individual is given by:

$$
\begin{gathered}
n_{k, t}^{q}=0, \\
n_{h, t}^{q}=\frac{\bar{n}}{\left(1+\theta_{l}\right)}, \\
l_{k, t}^{q}=0, \\
l_{h, t}^{q}=\frac{\theta_{l} \bar{n}}{\left(1+\theta_{l}\right)} .
\end{gathered}
$$

Importantly, we note that, in the absence of isolation, the optimal time allocation for infected individuals with a mild form of the illness would be the same as that of recovered people. This result is similar to the cases of ERTa and KUX in that infected people do not internalize the effect of their time allocation on the spread of the disease (and therefore the impact on the utility of the rest of the population). 
Severely ill infected individuals. The lifetime utility of an infected person who has developed severe symptoms, $U_{t}^{i}$, is given by

$$
U_{t}^{i}=u\left(c_{t}^{i}, l_{t}^{i}\right)+\beta\left[\pi_{r}^{i} U_{t+1}^{r}+\left(1-\pi_{r}^{i}-\pi_{r}^{d}\right) U_{t+1}^{i}\right]
$$

where, as is standard in macro and health economics, we assume that the cost of death is the foregone utility of life.

Importantly, the amount of time that a severely ill infected individual can devote to work and leisure falls from $\bar{n}$ to $\bar{n}^{i}$ and, as noted above, we assume that all infected individuals displaying severe symptoms are isolated at home: $n_{k, t}^{i}=l_{k, t}^{i}=0$.

In this case, it can be shown that the solution to the problem faced by severely ill individuals is the same as that of isolated infected individuals, once we account for the reduction in their available time from $\bar{n}$ to $\bar{n}^{i}$ :

$$
\begin{gathered}
n_{k, t}^{i}=0, \\
n_{h, t}^{i}=\frac{\bar{n}^{i}}{\left(1+\theta_{l}\right)}, \\
l_{k, t}^{i}=0, \\
l_{h, t}^{i}=\frac{\theta_{l} \bar{n}^{i}}{\left(1+\theta_{l}\right)} .
\end{gathered}
$$

\subsection{The choice of an individual with an unknown health status: the epidemic perceived by a behavioural agent}

\subsubsection{The lifetime utility of people with unknown health status.}

Under the assumption of the silent transmission of the disease, we have that susceptible individuals $(s)$, asymptomatic carriers $(a)$ and undetected recovered people $(z)$ do not know the health group to which they belong. Let $v=\{s, a, z\}$ denote the group of individuals who do not know their health status. However, these individuals know that they are not infected with severe symptoms and are not isolated or are certain that they have recovered (since, for the latter, they would need confirmation that they have been infected with the disease either by having shown severe symptoms or by having beeen isolated). Based on this information, they form time-t subjective probabilities about their health status. Specifically, let $\widetilde{p}\left(s_{t} \mid v_{t}\right), \widetilde{p}\left(a_{t} \mid v_{t}\right)$ and $\widetilde{p}\left(z_{t} \mid v_{t}\right)$ with $v=\{s, a, z\}$ denote the subjective probabilities of an individual who does not know their health status; i.e., whether they are susceptible, infected with mild symptoms or recovered, respectively.

Given these subjective probabilities, we can re-write the lifetime utility of an individual 
with an unknown health status as

$$
\begin{aligned}
\widetilde{U}_{t}^{v}= & u\left(c_{t}^{v}, l_{t}^{v}\right)+\beta\left[\widetilde{p}\left(i_{t+1} \mid v_{t}\right) u_{t+1}^{i}+\widetilde{p}\left(q_{t+1} \mid v_{t}\right) u_{t+1}^{q}+\widetilde{p}\left(v_{t+1} \mid v_{t}\right) u_{t+1}^{v}\right] \\
& +\beta^{2}\left[\widetilde{p}\left(i_{t+2} \mid v_{t}\right) U_{t+2}^{i}+\widetilde{p}\left(q_{t+2} \mid v_{t}\right) U_{t+2}^{q}+\widetilde{p}\left(r_{t+2} \mid v_{t}\right) U_{t+2}^{r}+\widetilde{p}\left(v_{t+2} \mid v_{t}\right) \widetilde{U}_{t+2}^{v}\right]
\end{aligned}
$$

where $u_{t+1}^{i} \equiv u\left(c_{t+1}^{i}, l_{t+1}^{i}\right), u_{t+1}^{q} \equiv u\left(c_{t+1}^{q}, l_{t+1}^{q}\right), u_{t+1}^{v} \equiv u\left(c_{t+1}^{v}, l_{t+1}^{v}\right)$ are the momentary utilities of infected individuals with severe symptoms, isolated individuals, and agents with unknown health status who are evaluated at their consumption and leisure decisions in $t+1 .^{14}$ Note that the perceived lifetime utility at time $t$ of a representative agent with unknown health status, $\widetilde{U}_{t}^{v}$, depends on $\widetilde{p}\left(j_{t+1} \mid v_{t}\right)$ and $\widetilde{p}\left(j_{t+2} \mid v_{t}\right)$ for $j=\{v, i, q, r, d\}$ : their subjective probabilities regarding their potential (observed) health status at time $t+1$ and $t+2$.

Importantly, as a main difference with ERTa and KUX, we do not necessarily assume that the subjective and objective probabilities coincide. Instead, we follow Gabaix (2020) in that, in our model, the agents do not necessarily understand the post-outbreak world nor how their behaviour might affect their probability of becoming infected). ${ }^{15}$ In the spirit of Gabaix (2020), we capture this mathematically by assuming that the agents, as they simulate the future, shrink their simulations toward the pre-epidemic steady state of the economy.

The epidemic perceived by the behavioural agent. We assume that the agent's perception of the probabilities that govern the population dynamics are

$$
\begin{aligned}
\tilde{\pi}_{l} & =\bar{m} \pi_{l}, \\
\tilde{\pi}_{n} & =\bar{m} \pi_{n}, \\
\tilde{\pi}_{h} & =\bar{m} \pi_{h}, \\
\tilde{\pi}_{i} & =\bar{m} \pi_{i}, \\
\tilde{\pi}_{d}^{i} & =\bar{m} \pi_{d}^{i}, \\
\tilde{\pi}_{r}^{i} & =(1-\bar{m})+\bar{m} \pi_{r}^{i}, \\
\tilde{\pi}_{r}^{a} & =(1-\bar{m})+\bar{m} \pi_{r}^{a},
\end{aligned}
$$

\footnotetext{
${ }^{14}$ Similar to ERTb, we assume that all individuals with unknown health status have the same subjective probabilities. Consequently, they choose the same time allocation.

${ }^{15}$ Gabaix (2020) presents a behavioural New Keynesian model in which the agents present partial myopia toward distant atypical events. See also Gabaix (2019) for an introduction to the literature of behavioural inattention.
} 
where $0 \leq \bar{m} \leq 1$ is a "cognitive discounting" parameter that measures the agent's attention to the epidemic. ${ }^{16}$ Consequently, susceptible agents perceive their probability of becoming infected as

$$
\begin{aligned}
\widetilde{\tau}_{t}= & \tilde{\pi}_{n} n_{k, t}^{v}\left(A_{t} n_{k, t}^{a}\right)+\widetilde{\pi}_{l} l_{k, t}^{v}\left(A_{t} l_{k, t}^{a}\right) \\
& +\widetilde{\pi}_{h}\left(n_{k, t}^{v}+l_{k, t}^{v}\right)\left(n_{h, t}^{v}+l_{h, t}^{v}\right)\left[A_{t}\left(n_{h, t}^{a}+l_{h, t}^{a}\right)\right] .
\end{aligned}
$$

When $\bar{m}=0$, the agents in the model are naïve and solely rely on their pre-epidemic perception of the world. Consequently, they do not change their behaviour given that they do not think they can be infected (i.e., $\widetilde{\tau}_{t}=0$ ). On the other hand, when $\bar{m}=1$, the agents are fully rational in that they are aware of the initial outbreak and understand the laws of motion governing the population health dynamics, and the agents' subjective perceptions of the world coincide with the objective laws of motion governing the population dynamics (i.e., $\widetilde{\tau}_{t}=\tau_{t}$ ).

When $0<\bar{m}<1$, the agents only partially understand the consequences of their actions. The agents start from their default perceptions of the world (in our case, the pre-epidemic steady state) and, after the outbreak, adjust them toward the new reality. However, when $0<\bar{m}<1$, this adjustment is not enough and results in agents that are over-optimistic:

1. They perceive the probability of becoming infected as being lower than in reality, $\tilde{\pi}_{l} \leq \pi_{l}, \tilde{\pi}_{n} \leq \pi_{n}$ and $\tilde{\pi}_{h} \leq \pi_{h}$ (equations 33, 34 and 35, respectively);

2. Conditional on being infected, they perceive the probability of developing severe symptoms as also being lower than in reality, $\tilde{\pi}_{i} \leq \pi_{i}$ (equation 36 );

3. Conditional on developing severe symptoms, they perceive the mortality rate as being lower, $\tilde{\pi}_{d}^{i} \leq \pi_{d}^{i}$ (equation 37 ), and the probability of recovery conditional on being infected as being higher than in reality, $\tilde{\pi}_{r}^{i} \geq \pi_{r}^{i}$ and $\widetilde{\pi}_{r}^{a} \geq \pi_{r}^{a}$ (equations 38 and 39 , respectively).

Consequently, this type of behavioural agent under-reacts to the outbreak and the evolution of the epidemic (if compared to a fully rational agent).

Given their perceived health population dynamics in equations (33)-(39) and their subjective beliefs over their time $t$ health status, agents form subjective probabilities over their potential health status at time $t+1$ and $t+2$ in equation (32).

\footnotetext{
${ }^{16}$ For now, we consider $\bar{m}$ to be constant. In Section 5.2 below, we analyze the case where there is time-varying inattention to the epidemic.
} 
Subjective probabilities about health status at $t+1$. Since only susceptible individuals can get infected, an individual an with unknown health status perceives the probability that they will become infected as the product of their perceived probability that a susceptible individual is infected, $\widetilde{\tau}_{t}$, and the perceived probability of being susceptible, $\widetilde{p}\left(s_{t} \mid v_{t}\right)$. Consequently, the perceived probability of remaining susceptible to the disease at time $t+1$, conditional on being an individual with unknown health status, is given by

$$
\widetilde{p}\left(s_{t+1} \mid v_{t}\right)=\left(1-\widetilde{\tau}_{t}\right) \widetilde{p}\left(s_{t} \mid v_{t}\right),
$$

However, an individual with unknown health status perceives the probability of being infected with severe symptoms at time $t+1$ as being

$$
\widetilde{p}\left(i_{t+1} \mid v_{t}\right)=\tilde{\pi}_{i} \widetilde{\tau}_{t} \tilde{p}\left(s_{t} \mid v_{t}\right)
$$

and perceives the probability of being mildly symptomatic and isolated at time $t+1$ as

$$
\widetilde{p}\left(q_{t+1} \mid v_{t}\right)=\alpha\left(1-\widetilde{\pi}_{i}\right) \widetilde{\tau} t \widetilde{p}\left(s_{t} \mid v_{t}\right)
$$

Finally, since it is not possible for an individual with an unknown health status at time $t$ to either die or fully recover at time $t+1$, we have that $\widetilde{p}\left(d_{t+1} \mid v_{t}\right)=\widetilde{p}\left(r_{t+1} \mid v_{t}\right)=0$ and, consequently,

$$
\widetilde{p}\left(v_{t+1} \mid v_{t}\right)=1-\widetilde{p}\left(i_{t+1} \mid v_{t}\right)-\widetilde{p}\left(q_{t+1} \mid v_{t}\right)
$$

Subjective probabilities about health status at $t+2$. Furthermore, agents need to form beliefs regarding their health status at time $t+2$ (cf. equation 32 above). We have that agents perceive their probability of becoming infected with severe symptoms at time $t+2$ conditional on being an individual with an unknown health status at time $t$ as

$$
\begin{aligned}
\widetilde{p}\left(i_{t+2} \mid v_{t}\right) & =\widetilde{p}\left(i_{t+2} \mid v_{t+1}\right) \widetilde{p}\left(v_{t+1} \mid v_{t}\right)+\widetilde{p}\left(i_{t+2} \mid i_{t+1}\right) \widetilde{p}\left(i_{t+1} \mid v_{t}\right) \\
& =\widetilde{\pi}_{i} \widetilde{\tau}_{t+1} \widetilde{p}\left(s_{t+1} \mid v_{t}\right)+\left(1-\widetilde{\pi}_{r}^{i}-\widetilde{\pi}_{d}^{i}\right) \widetilde{p}\left(i_{t+1} \mid v_{t}\right) \\
& =\widetilde{\pi}_{i} \widetilde{\tau}_{t+1}\left(1-\widetilde{\tau}_{t}\right) \widetilde{p}\left(s_{t} \mid v_{t}\right)+\left(1-\widetilde{\pi}_{r}^{i}-\widetilde{\pi}_{d}^{i}\right) \widetilde{\pi}_{i} \widetilde{\tau}_{t} \widetilde{p}\left(s_{t} \mid v_{t}\right),
\end{aligned}
$$

where we have that $\widetilde{p}\left(s_{t+1} \mid v_{t+1}\right)=\widetilde{p}\left(s_{t+1} \mid v_{t}\right) / \widetilde{p}\left(v_{t+1} \mid v_{t}\right), \widetilde{p}\left(i_{t+2} \mid i_{t+1}\right)=\left(1-\tilde{\pi}_{r}^{i}-\tilde{\pi}_{d}^{i}\right)$ and equations (41) and (42). The first term captures the subjective probability that an individual with an unknown health status at time $t$ that remained as such at time $t+1$ developed a severe form of the illness at time $t+2$. The second term captures the subjective probability that an individual with an unknown health status at time $t$ developed a severe form of the illness at time $t+1$, but did not recover at time $t+2$. 
Similarly, it is possible to show that the subjective probability of being mildly symptomatic and isolated at time $t+2$ conditional on being an individual with unknown health status at time $t$ is

$$
\begin{aligned}
\widetilde{p}\left(q_{t+2} \mid v_{t}\right) & =\widetilde{p}\left(q_{t+2} \mid v_{t+1}\right) \widetilde{p}\left(v_{t+1} \mid v_{t}\right)+\widetilde{p}\left(q_{t+2} \mid q_{t+1}\right) \widetilde{p}\left(q_{t+1} \mid v_{t}\right) \\
& =\alpha\left(1-\widetilde{\pi}_{i}\right) \widetilde{\tau}_{t+1}\left(1-\widetilde{\tau}_{t}\right) \widetilde{p}\left(s_{t} \mid v_{t}\right)+\left(1-\widetilde{\pi}_{r}^{a}\right) \alpha\left(1-\widetilde{\pi}_{i}\right) \widetilde{\tau}_{t} \widetilde{p}\left(s_{t} \mid v_{t}\right) .
\end{aligned}
$$

Again, the first term captures the subjective probability that an individual with an unknown health status at time $t$ that remained as such at time $t+1$ developed a mild form of the illness and is detected (and isolated) at time $t+2$. The second term captures the subjective probability that an individual with an unknown health status at time $t$ is quarantined at time $t+1$ but did not recover at time $t+2$ (and, therefore, remained isolated).

The subjective probability of being detected and recovered at time $t+2$ satisfies

$$
\begin{aligned}
\widetilde{p}\left(r_{t+2} \mid v_{t}\right) & =\widetilde{p}\left(r_{t+2} \mid i_{t+1}\right) \widetilde{p}\left(i_{t+1} \mid v_{t}\right)+\widetilde{p}\left(r_{t+2} \mid q_{t+1}\right) \widetilde{p}\left(q_{t+1} \mid v_{t}\right) \\
& =\widetilde{\pi}_{r}^{i} \widetilde{\pi}_{i} \widetilde{\tau}_{t} \widetilde{p}\left(s_{t} \mid v_{t}\right)+\widetilde{\pi}_{r}^{a} \alpha\left(1-\widetilde{\pi}_{i}\right) \widetilde{\tau}_{t} \widetilde{p}\left(s_{t} \mid v_{t}\right) .
\end{aligned}
$$

The first term captures the subjective probability that an individual with an unknown health status at time $t$ developed a severe form of the disease at time $t+1$ and subsequently recovered at time $t+2$. The second term captures the subjective probability that an individual with an unknown health status at time $t$ develops a mild version of the illness and is detected (and isolated) at time $t+1$ and subsequently recovers at time $t+2$.

Finally, the agent perceives their probability of dying at time $t+2$ as

$$
\widetilde{p}\left(d_{t+2} \mid v_{t}\right)=\widetilde{p}\left(d_{t+2} \mid i_{t+1}\right) \widetilde{p}\left(i_{t+1} \mid v_{t}\right)=\widetilde{\pi}_{d}^{i} \widetilde{\pi}_{i} \widetilde{\tau} t \widetilde{p}\left(s_{t} \mid v_{t}\right)
$$

and their probability of remaining with an unknown health status at $t+2$ as

$$
\widetilde{p}\left(v_{t+2} \mid v_{t}\right)=1-\widetilde{p}\left(i_{t+2} \mid v_{t}\right)-\widetilde{p}\left(q_{t+2} \mid v_{t}\right)-\widetilde{p}\left(r_{t+2} \mid v_{t}\right)-\widetilde{p}\left(d_{t+2} \mid v_{t}\right) .
$$

Subjective probabilities about current health status. Note that agents need to form beliefs over their probability of being susceptible, $\widetilde{p}\left(s_{t} \mid v_{t}\right)$, to form their beliefs regarding their future health status in equations (41)-(49). We assume that agents are only inattentive with respect to the future and that, consequently, the objective and subjective probabilities of being susceptible coincide: $\widetilde{p}\left(s_{t} \mid v_{t}\right)=\widetilde{p}\left(s_{t} \mid v_{t}\right)=S_{t} /\left(S_{t}+A_{t}+Z_{t}\right)$. 


\subsubsection{First-order conditions.}

A representative agent with an unknown health status maximizes the right-hand side of (32) subject to the time constraint (2), the budget constraints (3) and (4), the perceived disease transmission function in equation (40) and the perceived probabilities for the population health dynamics in equations (41) to (49).

The first-order conditions of an agent with an unknown health status with respect to $c_{h, t}^{v}$ and $c_{k, t}^{v}$ are

$$
\begin{gathered}
\omega_{h}^{c}\left(c_{t}^{v}\right)^{-1}\left(\frac{c_{t}^{v}}{c_{h, t}^{v}}\right)^{1 / \eta}-\lambda_{b h, t}^{v}=0, \\
\left(c_{t}^{v}\right)^{-1}\left(\frac{c_{t}}{c_{k, t}^{v}}\right)^{1 / \eta}-\lambda_{b k, t}^{v}=0,
\end{gathered}
$$

where $\lambda_{b h, t}^{v}$, and $\lambda_{b k, t}^{v}$ are the Lagrange multipliers that are associated with constraints (3) and (4), respectively. We note that equations (50) and (51) coincide with those of the representative agent in the pre-infection economy.

The first-order conditions with respect to $l_{h, t}^{v}, l_{k, t}^{v}$ and $n_{h, t}^{v}$ and $n_{k, t}^{v}$ are:

$$
\begin{gathered}
\theta_{l} \omega_{h}^{l}\left(l_{t}^{v}\right)^{-1}\left(\frac{l_{t}^{v}}{l_{h, t}^{v}}\right)^{1 / \sigma}+\lambda_{a, t}^{v}+\lambda_{\widetilde{\tau}, t}^{v} \widetilde{\pi}_{h}\left(n_{k, t}^{v}+l_{k, t}^{v}\right)\left[A_{t}\left(n_{h, t}^{a}+l_{h, t}^{a}\right)\right]=0, \\
\theta_{l}\left(l_{t}^{v}\right)^{-1}\left(\frac{l_{t}^{v}}{l_{k, t}^{v}}\right)^{1 / \sigma}+\lambda_{a, t}^{v}+\lambda_{\widetilde{\tau}, t}^{v} \widetilde{\pi}_{l}\left(A_{t} l_{k, t}^{a}\right)+\lambda_{\widetilde{\tau}, t}^{v} \widetilde{\pi}_{h}\left(n_{h, t}^{v}+l_{h, t}^{v}\right)\left[A_{t}\left(n_{h, t}^{a}+l_{h, t}^{a}\right)\right]=0, \\
\lambda_{a, t}^{v}+A_{h} \lambda_{b h, t}^{v}+\lambda_{\widetilde{\tau}, t}^{v} \widetilde{\pi}_{h}\left(n_{k, t}^{v}+l_{k, t}^{v}\right)\left[A_{t}\left(n_{h, t}^{a}+l_{h, t}^{a}\right)\right]=0, \\
\lambda_{a, t}^{v}+A_{k} \lambda_{b k, t}^{v}+\lambda_{\widetilde{\tau}, t}^{v} \widetilde{\pi}_{n}\left(A_{t} n_{k, t}^{a}\right)+\lambda_{\widetilde{\tau}, t}^{v} \widetilde{\pi}_{h}\left(n_{h, t}^{v}+l_{h, t}^{v}\right)\left[A_{t}\left(n_{h, t}^{a}+l_{h, t}^{a}\right)\right]=0,
\end{gathered}
$$

where $\lambda_{a, t}^{v}$ is the Lagrange multiplier that is associated with the time allocation constraint in (2), and $\lambda_{\widetilde{\tau}, t}$ is the Lagrange multiplier that is associated with the constraint that defines the perceived probability of becoming infected in equation (40).

Note that equations (52) to (55) coincide with those of the representative agent in the pre-infection economy when $\bar{m}=0$ (since, in that case, we have that $\widetilde{\pi}_{n}=\widetilde{\pi}_{l}=\widetilde{\pi}_{h}=0$ ). That is, individuals who perceive that the probability of becoming infected is not affected by their decisions will, consequently, choose their pre-infection time allocation.

Finally, the first-order condition with respect to $\widetilde{\tau}_{t}$ is given by

$$
\begin{aligned}
& \lambda_{\widetilde{\tau}, t}^{v}-\beta\left[\frac{\partial \widetilde{p}\left(i_{t+1} \mid v_{t}\right)}{\partial \widetilde{\tau}_{t}} u_{t+1}^{i}+\frac{\partial \widetilde{p}\left(q_{t+1} \mid v_{t}\right)}{\partial \widetilde{\tau}_{t}} u_{t+1}^{q}+\frac{\partial \widetilde{p}\left(v_{t+1} \mid v_{t}\right)}{\partial \widetilde{\tau}_{t}} u_{t+1}^{v}\right] \\
& -\beta^{2}\left[\frac{\partial \widetilde{p}\left(i_{t+2} \mid v_{t}\right)}{\partial \widetilde{\tau}_{t}} U_{t+2}^{i}+\frac{\partial \widetilde{p}\left(q_{t+2} \mid v_{t}\right)}{\partial \widetilde{\tau}_{t}} U_{t+2}^{q}+\frac{\partial \widetilde{p}\left(r_{t+2} \mid v_{t}\right)}{\partial \widetilde{\tau}_{t}} U_{t+2}^{r}+\frac{\partial \widetilde{p}\left(v_{t+2} \mid v_{t}\right)}{\partial \widetilde{\tau}_{t}} U_{t+2}^{v}\right]
\end{aligned}
$$

where the corresponding derivatives of equations (41)-(49) can be found in the appendix. 


\subsection{Computational strategy}

We solve the model numerically using a similar approach to that in ERTa. Specifically, we guess the path for $\left\{n_{h, t}^{u}, n_{k, t}^{u}, l_{h, t}^{u}\right\}_{t=0}^{H-1}$ for some large horizon which, in our case, we choose to be $H=1000$ weeks (20 years) and assume that the model reaches the new steady state by then. Given this path, we compute the sequence of the remaining unknown variables and using a gradient-based method, we adjust the guesses $\left\{n_{h, t}^{u}, n_{k, t}^{u}, l_{h, t}^{u}\right\}_{t=0}^{H-1}$ so that the first-order conditions of an individual with an unknown health status hold with arbitrary precision.

We note, however, that while arbitrarily close, there are cases in which the model has not converged to its steady state by week $H=1000$. We advance that this is related to the fact that the behaviour of the individuals in this economy is such that the epidemic grows linearly and, consequently, it takes a very long time for the epidemic to die out on its own (i.e., for enough people to be infected such that it is difficult for the disease to find new susceptible hosts). We solve this issue by assuming that a vaccine for the disease is discovered at the beginning of period $H$ and that all individuals with unknown health status are vaccinated (and consequently, they are all considered recovered in period $H$ ). ${ }^{17}$

\subsection{The disease reproduction numbers}

A key statistic that gauges a disease's contagiousness is the basic reproduction number of an infection, $\mathcal{R}_{0}$, which is defined as the total expected number of cases that are directly generated by one infected person (with measure zero) in a population where all individuals are susceptible to infection $\left(S_{t}=1\right)$ and that is absent of any deliberate intervention to slow the disease (like quarantines or isolation). Importantly, if $\mathcal{R}_{0}>1$, then an arbitrarily small initial infection outbreak will spread exponentially until limited by a fraction of the susceptible individuals in the population that is sufficiently low.

Let $\gamma_{0}$ be the average rate of infection at time $t=0$, evaluated at the pre-epidemic steady-state time allocation and under the assumption that no infected individual (either with mild or severe symptoms) is isolated:

$$
\gamma_{0}=\pi_{n}\left(n_{k, t}^{r}\right)^{2}+\pi_{l}\left(l_{k, t}^{r}\right)^{2}+\pi_{h}\left(n_{k, t}^{r}+l_{k, t}^{r}\right)\left(n_{h, t}^{r}+l_{h, t}^{r}\right)^{2}
$$

where we have $\gamma_{0}=T_{0} /\left(I_{0}+A_{0}+Q_{0}\right)$, with $T_{0}$ being the number of newly infected people

\footnotetext{
${ }^{17}$ We note that this approach does not change the optimal allocation of the agents with respect to simulations with a larger value for $H$; the exception is for (approximately) the last four weeks of the simulation (where the agent's time allocation starts to quickly converge to the new steady state as the deployment of the vaccine is within reach.
} 
when the agents choose the pre-infection steady-state time allocation, and $I_{0}=\pi_{i} \varepsilon$, $A_{0}=\left(1-\pi_{i}\right) \varepsilon, Q_{0}=0, S_{0}=1-\varepsilon$, where we have taken the limit of $\varepsilon$ to zero. Consequently, the expected number of infections caused by a single infected person at time $t=0$ is given by

$$
\mathcal{R}_{0}=\sum_{t=0}^{\infty} \gamma_{0}\left[1-\pi_{i}\left(\pi_{r}^{i}+\pi_{d}^{i}\right)-\left(1-\pi_{i}\right) \pi_{r}^{a}\right]^{t}=\frac{\gamma_{0}}{\pi_{r}^{a}},
$$

where $\left[1-\pi_{i}\left(\pi_{r}^{i}+\pi_{d}^{i}\right)-\left(1-\pi_{i}\right) \pi_{d}^{a}\right]^{t}$ is the probability that the (average) infected person reaches period $t$ without recovering or dying, and where the last equality comes from our assumption that $\pi_{r}^{a}=\pi_{r}^{i}+\pi_{d}^{i}$ in our model.

The effective reproduction number, $\mathcal{R}_{t}^{e}$, is defined as the total expected number of cases that are directly generated by one infected person (again, with measure zero) in the current state of the population (as opposed to a population where all individuals are susceptible to infection):

$$
\mathcal{R}_{t}^{e}=\frac{\gamma_{t}}{\pi_{r}^{a}}
$$

where $\gamma_{t}=T_{t} /\left(I_{t}+A_{t}+Q_{t}\right)$ is the actual average rate of infection at time $t$ (i.e., the ratio of the number of newly infected individuals to the total number of infected). Note that if $\mathcal{R}_{t}^{e}>1$, then the number of infected individuals grows exponentially, while $\mathcal{R}_{t}^{e}<1$ indicates that the number of cases is declining and that the epidemic is dying out. When $\mathcal{R}_{t}^{e}=1$, we have that $\gamma_{t}=\pi_{i}\left(\pi_{r}^{i}+\pi_{d}^{i}\right)-\left(1-\pi_{i}\right) \pi_{r}^{a}=\pi_{r}^{a}$. That is, for every person who recovers or dies, a new person gets infected, and the epidemic grows linearly where the rate of growth depends on the prevalence of the disease in the population. ${ }^{18}$

\section{Calibration}

We now report our choice of parameters, which we summarize in Table 1.

The pre-epidemic parameters. We set the elasticities of substitution for home versus market consumption and home versus social leisure to be the same and equal to three, $\eta=\sigma=3$. This is consistent with some of the analyses in KUX and the view that it is difficult to substitute at-home and outside activities.

As in ERTa, we note that our calibration requires a number of judgment calls. Specifically, we calibrate the parameters $\theta_{l}, \omega_{h}^{c}$, and $\omega_{h}^{l}$, such that in the pre-epidemic steady

\footnotetext{
${ }^{18}$ We note that, in our model, the effective reproduction number at time $t=0$ is not necessarily equal to the basic reproduction number, i.e. $\mathcal{R}_{0}^{e} \neq \mathcal{R}_{0}$, because, upon the initial outbreak, the agents change their behaviour at $t=0$ and we assume that all infected individuals with severe symptoms are isolated.
} 
state a representative person devotes 40 per cent of their time to production of the market good, 20 per cent of their time to home production, 10 per cent to social leisure and 30 per cent to home leisure. These time shares are estimated from the U.S. Bureau of Labor Statistics 2018 American Time Use Survey and are based on a broad interpretation of the activities in our model. For example, in the spirit of Becker (1965) and Gronau (1977), we interpret the term "working time" as the time needed to consume one unit of either the market or home good. As such, we compute the fraction of time working in the market as the sum of the time spent in the "working and work-related" and "educational activities" categories listed in the 2018 American Time Use Survey, but we also add the time spent "purchasing goods and services" and "eating and drinking outside of the house" (which, following ERTa, we estimate by multiplying the time spent "eating and drinking" by 54 per cent, which, according to the U.S. Department of Agriculture, is the fraction of total food expenditures on "food away from home" in 2018).

To compute the fraction of time devoted to home production, we add the time spent in "household activities;" time devoted to "caring for and helping household and nonhousehold members;" time spent on "telephone calls, mail, and e-mail" and time spent "eating and drinking inside the house," which are also categories of the American 2018 Time Use Survey.

We also interpret the activity of "social leisure" very broadly in order to capture the potential transmission in the general community that is not linked to economic decisions. For this reason, we compute the fraction of time devoted to "social leisure" as the sum of time spent in "organizational, civic, and religious activities;" time spent "socializing and communicating" and half of the time spent "participating in sports, exercise, and recreation" and "other leisure and sports activities, including travel." ${ }^{19}$ Finally, we compute the fraction of time devoted to "home leisure" as the sum of the time spent "watching television," "reading," "playing games and computer use for leisure" and the other fifty per cent of the time spent on "participating in sports, exercise, and recreation" and "other leisure and sports activities, including travel."

Similar to ERTa, we calibrate $A_{k}$ such that, in the pre-epidemic steady state, the representative agent earns a weekly income of $\$ 58,000 / 52$, which coincides with the percapita income in 2019 from the U.S. Bureau of Economic Analysis. We choose $A_{h}$ such that the value of home production is one fourth of the market production, which is consistent

\footnotetext{
${ }^{19}$ Since there is no breakdown of team versus individual sports or exercise and recreation activities, we make the call of dividing the time spent "participating in sports, exercise, and recreation" and "other leisure and sports activities, including travel" evenly across time spent at home and social leisure.
} 
with the U.S. Bureau of Economic Analysis estimates of the value of household production (see Kanal and Kornegay, 2019).

Finally, we set $\beta=0.96^{1 / 52}$ as in ERTa and KUX and choose $\theta_{0}$ such that the value of a human life is US\$ 3.5 million as in Hall, Jones and Klenow (2020).

The epidemic parameters. We set the probability of developing a severe form of the illness to be $\pi_{i}=0.25$. This choice is consistent with Poletti et al. (2020), who use data on contacts of the COVID-19 index cases detected in Lombardy and find that 75 per cent infected individuals younger than 60 years of age but older than 20 years do not show recognizable symptoms (this being respiratory symptoms such as a cough or shortness of breath, or a fever higher than $37.5 \mathrm{C})$.

Consistent with ERTb, and the fact that our model is designed to capture the decisions of working-age individuals, we assume a 0.2 per cent mortality rate. This implies, under our assumption that the mortality rate conditional on developing the mild form of the disease is zero, a mortality rate conditional on developing the severe form of the disease of 0.8 per cent. Further, we follow these authors in assuming that it takes, on average, 14 days to either die or recover from the disease. Since our model is weekly, we have that $\pi_{r}^{a}=\pi_{r}^{i}+\pi_{d}^{i}=7 / 14$ and $\pi_{d}^{i}=7 \times 0.008 / 14$. Further, we assume that the severe form of the disease reduces by a third the time available for working and leisure.

We calibrate $\pi_{n}, \pi_{l}$ and $\pi_{h}$ in a similar fashion to ERTa. Specifically, we pin down the values for two of these three parameters by assuming that the steady-state shares of the three infection channels in our model are the same:

$$
\begin{gathered}
\frac{\pi_{n}\left(n_{k, t}^{r}\right)^{2}}{\pi_{n}\left(n_{k, t}^{r}\right)^{2}+\pi_{l}\left(l_{k, t}^{r}\right)^{2}+\pi_{h}\left(n_{k, t}^{r}+l_{k, t}^{r}\right)\left(n_{h, t}^{r}+l_{h, t}^{r}\right)^{2}}=\frac{1}{3}, \\
\frac{\pi_{l}\left(l_{k, t}^{r}\right)^{2}}{\pi_{n}\left(n_{k, t}^{r}\right)^{2}+\pi_{l}\left(l_{k, t}^{r}\right)^{2}+\pi_{h}\left(n_{k, t}^{r}+l_{k, t}^{r}\right)\left(n_{h, t}^{r}+l_{h, t}^{r}\right)^{2}}=\frac{1}{3} .
\end{gathered}
$$

Such an assumption is consistent with Ferguson et al. (2006), who find that influenza transmissions that occur in schools and workplaces, in the general community and in the household occur at roughly the same rate (37, 33 and 30 per cent, respectively). We pin down the third parameter by assuming that the basic reproduction number, $\mathcal{R}_{0}$, for COVID-19 is equal to 2.5. This assumption is consistent with the point estimate of 2.38 and the 95 per cent confidence interval from 2.04 to 2.77 reported in Li et al. (2020) and higher than the assumed value of 1.5 in ERTa. ${ }^{20}$

\footnotetext{
${ }^{20}$ Rather than directly assuming a specific value for the basic reproduction rate, ERT assumes that,
} 
Finally, we normalize the initial population to one and assume that the size of the initial outbreak, $\varepsilon$, is 0.01 per cent. Our outbreak is slightly smaller than in ERTa to compensate for the higher assumed $\mathcal{R}_{0}$ for COVID-19.

\section{Benchmark results}

\subsection{Results for a model with naïve agents}

Figure 2A shows the equilibrium population dynamics implied by a model with naïve agents that do not change their allocation of time in response to the outbreak (i.e., $\bar{m}=$ 0 ). In such a model, the probability of transmission is constant and, therefore, these population dynamics are equivalent to those of an SIR-type version of our model.

Specifically, the solid lines in Figure 2A display the population dynamics when there is no detection and isolation of mildly symptomatic infected individuals $(\alpha=0)$. In this case, the share of the initial population that is infected with severe symptoms peaks at 3.66 per cent in week 25. By then, approximately 50 per cent of the population has already been infected. However, given that a large fraction of the population remains infectious at the peak (10.97 per cent of the population is infected with a mild form of the illness at that time), the disease continues to spread for a longer period despite the effective reproduction number, $\mathcal{R}_{t}^{e}$, becoming less than one. Eventually, 80 per cent of the population becomes infected and 0.15 per cent of the population dies, which, assuming a U.S. (Canadian) population of 330 million (38 million), implies that the disease kills roughly half a million $(55,000)$ people. ${ }^{21}$

In our model, detection and isolation policies improve the outcome of the epidemic. Specifically, detecting and isolating 20 per cent of new mildly symptomatic cases (dotdashed lines in Figure 2A) (i) reduces the peak of severely infected individuals from 3.66 to 1.69 per cent, (ii) reduces the peak of mildly symptomatic infected individuals from 10.97 to 4.05, (iii) delays the peak of the epidemic from week 25 to week 36, (iv) reduces the final size of the epidemic from 80 per cent to 60 per cent, and (v) reduces the fraction of the population that dies from 0.15 per cent to 0.12 per cent. That is, increasing the detection rate from 0 per cent to 20 per cent would save approximately 100,000 lives in the case of the U.S. and 11,000 lives in the case of Canada.

in the limit, 60 per cent of the population either recovers or dies. We note, using the results in Miller (2012) on the final size of epidemics, this can be mapped back to a basic reproduction rate of 1.5.

${ }^{21}$ We note that the effective reproduction number at time $t=0$ is lower than 2.5 due to the assumption that all infected individuals with severe symptoms are detected and isolated. 
Moreover, further lives can be saved by increasing the detection/isolation rate of mildly symptomatic individuals. Increasing $\alpha$ to 40 per cent saves approximately 365,000 lives in the case of the U.S. and 40,000 in the case of Canada (dashed lines in Figure 2A), while a further increase to 80 per cent essentially reverses the epidemic (dotted lines in Figure $2 \mathrm{~A})$.

Figure 2B shows the time allocation of a naïve agent who does not know their health status. In this case, all four lines lie on top of each other as agents choose their preepidemic steady-state time allocation: 40 per cent is devoted to market production, 20 per cent to home production, 10 per cent to social leisure and 30 per cent to home leisure.

Similar to the results in ERTa, the epidemic induces a recession even if individuals do not change their behaviour. For example, consumption of the market good falls by approximately 3.5 per cent from their pre-epidemic steady-state value at the bottom of the recession (solid line in Figure 2C) when there is no detection and isolation of mildly symptomatic infected individuals $(\alpha=0)$. Moreover, even if the consumption of the home good increases during the epidemic, this does not compensate for the decline in the consumption of the market good. In fact, aggregate consumption (cf. equation 6 ) falls by 2.5 per cent from the pre-epidemic steady state at the bottom of the recession.

There are two main reasons for this fall in aggregate consumption. First, the size of the workforce falls over time as some severely ill individuals die. Second, infected individuals who develop a severe form of the illness are faced with less time for work and can only work in the home sector (which we assume to be less productive). The severity of the recession and of the epidemic are thus closely linked. For this reason, the severity of the recession can also be reduced through detection and isolation policies. For example, the dot-dashed (dashed) lines in Figure 2C show that detecting and isolating 20 per cent (40 per cent) of new mildly symptomatic cases reduces the peak decline in the consumption of the market good from approximately 3.5 per cent to roughly 2.5 per cent ( 0.5 per cent), reduces the peak decline in the consumption of the market and home good bundle from 2.5 per cent to roughly 1.5 per cent ( 0.25 per cent) and delays the trough of the recession. Further increasing $\alpha$ to 80 per cent essentially reverses the recession. These results suggest that increasing the detection/isolation rate of mildly symptomatic individuals flattens not only the infection curve but also the recession. 


\subsection{Results for a model with rational agents}

So far we have assumed that agents are naïve and therefore do not change their behaviour when faced with an epidemic (cf. Figure 2B). However, one would expect individuals to try to reduce the probability of being infected by, at least, partially reducing the time they spend in activities with a higher infection risk. For this reason, we now analyze the case where the agents are rational $(\bar{m}=1)$. The equilibrium population dynamics implied by the model are presented in Figure 3A.

Similar to the results in ERTa and KUX, individual rational behaviour is a strong mitigator of the impact of the epidemic on the health population dynamics. The reader can see this by noting the change in the scale of the graphs that show the population dynamics for the model with a rational agent (Figure 3A) versus the scale of the figures of the model with a naïve agent (Figure 2A). For example, we find that, in a model with naïve agents and no detection and isolation policies, the share of the initial population that is infected with severe (mild) symptoms peaks at 3.66 (10.97) per cent in week 25 and it quickly declines afterward. In contrast, in a model with rational agents (solid lines in Figure 3A), the fraction of severely (mildly) ill infected individuals peaks at 0.03 (0.08) per cent also in week 25. Similarly, we see that in a model with rational agents, when compared with a model with naïve agents, a substantially smaller fraction of the population becomes infected. For example, when $\alpha=0$, only roughly 10 per cent of the population becomes infected by the end of the third year compared with almost 80 per cent of the population by week 35 in the case of the model with naïve agents. Similarly, we see a substantial fall in the number of deaths: 0.02 per cent of the population when the agents are rational versus 0.15 per cent in the case where the agents are naïve.

In addition, there is also an important and noticeable difference between the dynamics of the effective reproduction rate, $\mathcal{R}_{t}^{e}$, in a model with naïve agents and those in a model with rational agents. In the case of the model with naïve agents (and $\alpha=0$ ), the effective reproduction rate is close to two at the beginning of the epidemic, where it remains for approximately 10 weeks. Then, the epidemic gathers momentum and, as the number of infected individuals increases, $\mathcal{R}_{t}^{e}$ starts falling very quickly and becomes less than 1 by week 25. Finally, the effective reproduction rate of the disease stabilizes at around 0.4 by week 40 . When the agents are rational, they already change their behaviour upon the initial outbreak in a way that makes $\mathcal{R}_{t}^{e}$ fall from 1.7 to 1 by week 25 , where it remains. That is, rational agents behave in a way that mitigates the explosive behaviour of an epidemic that is characterized by an effective reproduction rate above 1 . 
However, their behaviour does not drive the effective reproduction number below 1 and, consequently, does not eliminate the disease. Consequently, we find that, in our model, rational behaviour leads to an epidemic that grows linearly.

In Figure 3B, we can take a deeper look at the behaviour of the rational agent that leads to the effective reproduction rate stabilizing at around 1. Again, we continue to analyze the case of no detection and isolation policies (solid lines in Figure 3B) and discuss the implications of an increase in the detection and isolation rate later in this section.

When faced with the initial outbreak, the rational agent starts reallocating their time from market production to home production and from social leisure to at-home leisure. By week 25, the time worked in the market bottoms at 24 per cent (from a steady-state allocation of 40 per cent), the time devoted to home production peaks at 33 per cent (versus 20 per cent in steady state), the time devoted to social leisure falls to 3 per cent (versus 10 per cent in steady state) and the time devoted to home leisure increases to 40 per cent (versus 30 per cent in steady state). In other words, the rational agents partially self-isolate by reallocating most of their time to home activities. After week 25, these agents start increasing the time they devote to out-of-the-house activities albeit at a very slow pace that is consistent with keeping the reproduction rate at 1 . For example, by the end of the third year of the epidemic, the time allocated to market production and social leisure is still only 27 and 4 per cent, respectively. Moreover, the agents' time allocations to market production and social leisure are still 33 and 5 per cent, respectively (unreported in Figure 3B for the sake of space). That is, the economy has not returned to its new steady state by year ten after the outbreak.

Our results are similar to those in KUX. However, in contrast to these authors, we find that this endogenous reallocation of time has an important economic cost due to the home production sector having lower productivity than the market sector. For example, in the case of an economy with rational agents and no isolation of mildly symptomatic individuals, the consumption of the market good falls by 40 per cent of the pre-epidemic steady state by week 25 (solid lines in Figure 3C). Further, consumption is still 35 per cent (17 per cent) lower than such a steady state three (ten) years after the outbreak. We also note that, even though the number of hours devoted to home production substantially increase (with a corresponding increase in the consumption of the home good), the consumption of the basket of home and market goods (i.e., the aggregate consumption) also shows a substantial and persistent fall. In this case, consumption falls by roughly 
9 per cent from its pre-epidemic steady-state value by week 25 and is still 7 per cent (3 per cent) lower than such a steady state three (ten) years after the outbreak. Overall, the rational agents' response to the epidemic induces a substantial and persistent fall in aggregate consumption.

As in the case of the model with naïve agents, the severity of the recession can also be lessened by detecting and isolating mildly symptomatic infected individuals. Increasing the detection and isolation rate, $\alpha$, has two important benefits for mitigating the epidemic's effects on the economy. First, by reducing the pool of infected people who can spread the disease, increasing $\alpha$ allows the rational agents to spend more time in out-ofthe-house activities while still keeping the effective reproduction rate of the disease at 1 . Second, it allows a reduction in the number of undetected recovered individuals who are still reducing their consumption (since they are still assigning a positive probability to be susceptible) but who would return to their pre-epidemic allocation of time if they knew they had recovered. We note that the fraction of the population that does not know they have recovered amounts to 2 per cent by the end of the first year, 6 per cent by the end of the third year, and 18 per cent by end of year ten.

Increasing the detection rate to 20 per cent reduces the peak fall in aggregate consumption from roughly 9 per cent to approximately 4 per cent. This is related to the fact that the time devoted to market production falls to only $31 \%$ at the bottom of the recession (versus $24 \%$ when $\alpha=0$ ). Further increases in the detection rate of mildly symptomatic individuals to $\alpha=0.40$ almost reverses the epidemic's effects on the economy: agents reallocate only some of their social leisure to home leisure. With $\alpha=0.80$, agents are able to, essentially, return to their pre-epidemic behaviour.

Individual behaviour as an endogenous epidemic mitigation mechanism relies on the agents fully understanding the dangers of exponentially growing epidemics and, consequently, changing their behaviour so that the effective reproduction rate stabilizes at 1 . Therefore, deviations from the rational-expectations behaviour might lead to very different outcomes. We explore this in the next section.

\section{$5 \quad$ Results with behavioural agents}

\subsection{Results for a model with optimistic agents}

We now explore the outcomes of an epidemic where the agents do not fully understand the post-outbreak world. As noted above, we capture this mathematically by assuming that 
the agents, as they simulate the future, shrink their simulations toward the pre-epidemic steady state of the economy. Specifically, we adopt a value of the "cognitive discounting" parameter of $\bar{m}=1 / 2$. That is, the agents are optimistic in that they believe the disease is only half as deadly as the actual figure.

Figure $4 \mathrm{~A}$ displays the equilibrium population dynamics for the model where the agents are optimistic. Note that when $\alpha=0$, the share of the initial population that is infected with severe (mild) symptoms peaks at 0.11 per cent (0.33 per cent) in week 26 . This is a larger share of the population than in the model where the agents are rational but substantially smaller than in the case where agents are completely naïve and do not change their behaviour (please note, again, the change of scale in Figures 3A, 4A and $5 \mathrm{~A})$.

Interestingly, the effective reproduction rate of the disease stabilizes at 1 as in the model with the rational agents. That is, our behavioural agents still understand the dangers of an exponentially growing disease. However, by misperceiving the dynamics of the disease, an optimistic agent under-reacts to the epidemic. We show this in Figure 4B where we display the time allocation of the agent with an unknown health status. Specifically, we have that, by week 5 (10) after the outbreak, a rational agent has already reduced their time devoted to market production from 40 per cent to 37 per cent (31 per cent), while an optimistic agent is still working in the market 39.5 per cent (36 per cent) of their available time.

This delayed response results in a larger fraction of the population being infected in the initial phase of the epidemic. For example, when the agents are rational, only 1 per cent of the population becomes infected by week 25 . However, when the agents are optimistic, roughly 3 per cent of the population becomes infected by this point. Further, this higher prevalence leads to the epidemic having a much bigger impact in the medium run. By the end of the third year of the epidemic, 26 per cent of the population has been infected with the disease. We note that this occurs despite the reproduction rate stabilizing at 1 , given that linear growth starts from a higher prevalence of the disease (when compared to the model with the rational agents).

The reallocation of time being induced by the epidemic implies a decline in the consumption of the market good that is similar in magnitude to that of the case with the rational agents: approximately 40 per cent of the pre-epidemic steady state (Figure 4C). Similarly, the decline in aggregate consumption is approximately 8 per cent.

Finally, we note that, once again, detection and isolation policies can attenuate the 
outcomes of the epidemic. For example, detecting and isolating 40 per cent of mildly symptomatic infected people practically reverses both the health and economic outcomes of the epidemic.

\subsection{Results for a model with time-varying inattention}

In the previous section, we assume that the agents do not change the amount of attention they pay to the epidemic. We now analyze the implications of a model in which the agents are initially inattentive to the outbreak but over time become more attentive. Such behaviour is consistent with the findings of Davis et al. (2020) that the SARSCoV-2 virus causing COVID-19 was already spreading undetected in the United States in January and February 2020. ${ }^{22}$

Specifically, we assume the following functional form for the cognitive parameter:

$$
\bar{m}_{t}=\frac{\exp \left(m_{0}+m_{1} \sum_{j=0}^{11} \pi_{d}^{i} I_{t-j}+m_{2} t\right)}{1-\exp \left(m_{0}+m_{1} \sum_{j=0}^{11} \pi_{d}^{i} I_{t-j}+m_{2} t\right)}
$$

where $\sum_{j=0}^{11} \pi_{d}^{i} I_{t-j}$ captures the number of new deaths in the last 12 weeks ( 3 months). That is, the agents pay more attention when the number of deaths is high (similar to the behavioural model of Cochrane (2020)) and as time goes by (a reduced-form proxy for learning). The set of parameters $\left(m_{0}, m_{1}, m_{2}\right)^{\prime}$ are chosen such that (i) $\bar{m}_{t}=0$ for $t=0$ and zero deaths, (ii) $\bar{m}_{t}=0.99$ if quarterly new deaths were 200 per million people and $t=0$ and (iii) $\bar{m}_{t}=0.99$ if $t=100$ (two years) with zero deaths.

We can see in Figure 5A that a model where the agents display time-varying inattention generates epidemic waves. For example, when there is no detection and isolation of mildly symptomatic individuals, there is a first wave in which the share of the population that is infected with severe (mild) symptoms peaks at 0.33 per cent (1.07 per cent) at week 16 and there is a second smaller wave in which the share of the population that is infected with severe (mild) symptoms peaks at 0.11 per cent ( 0.32 per cent) at week $35 .{ }^{23}$

Such waves are also evident in the dynamics of the effective reproduction rate and the time allocation of the agent with an unknown health status. At first, the agents do not pay attention to the outbreak and, consequently, do not alter their time allocation (see Figure 5B). Consequently, the disease spreads unchecked. By the time the number of deaths sufficiently increases, and consequently the agent starts to pay attention, the

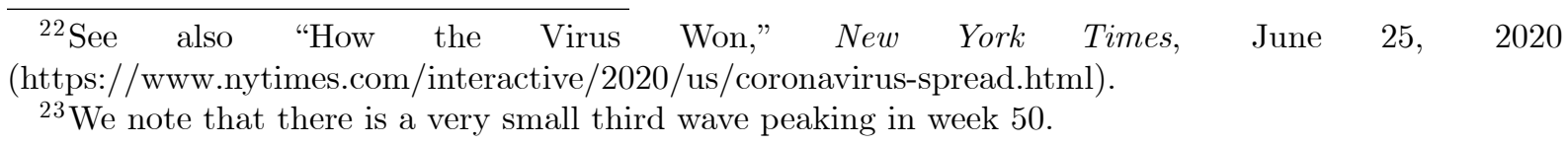


only option for the agent to slow down the disease is to essentially shelter at home. For example, the amount of time devoted to market production and social leisure bottoms at 5.5 per cent and 1 per cent, respectively, which makes the reproduction rate fall to 0.27 , thus, momentarily halting the spread of the disease.

However, as the number of deaths falls, the agents return to being inattentive and, consequently, are to quick to increase the time devoted to out-of-the-house activities. In particular, the time devoted to market production and social leisure increases, respectively, to 35 per cent and 6 per cent by week 30, making the effective reproduction rate jump to 1.34 and creating a second wave that peaks in week 35 .

Looking at the consumption dynamics in a model with time-varying inattention, we find that the epidemic waves also create consumption cycles (Figure 5C). Importantly, the consumption loss due to the lack of attention to the initial outbreak is dramatic: aggregate consumption falls 28 per cent from the pre-epidemic steady state by week 16 and, after recovering somewhat for a couple of weeks, it falls again to 12 per cent from the steady state. Given the agents' preference for smoothing their consumption stream, this cyclicality in consumption is very costly from a utility perspective.

Finally, we find once more that the effects of the epidemic on the agents' consumption profile could have been minimized by an increase in the rate of detection and isolation of mildly symptomatic infected individuals. In fact, detecting and isolating 40 per cent of mildly symptomatic individuals is enough to reverse the epidemic's effects on the economy.

\section{Final remarks}

In this paper, we studied the interaction between epidemics and economic decisions in a model where there is silent transmission of a disease and where (1) the agents allocate their time to market and home production and social and home leisure, (2) these activities differ in their degree of contagiousness, (3) some infected individuals are indistinguishable from susceptible individuals and (4) the agents are not necessarily rational.

Our results, based on a calibrated version of the model, suggest that supplementing symptom-based isolation with detection and isolation policies targeted at asymptomatic or mildly symptomatic cases can have a strong impact on reducing the severity of the recession that is induced by the COVID-19 pandemic. 


\section{References}

[1] Acemoglu, D., V. Chernozhukov, I. Werning and M.D. Whinston. 2020. "Optimal Targeted Lockdowns in a Multi-Group SIR Model.” NBER Working Paper No. 27102.

[2] Allcott, H., B.B. Lockwood and D. Taubinsky. 2019. "A Theory of Regressive Sin Taxes, with an Application to the Optimal Soda Tax." Quarterly Journal of Economics, 143: 1557-1626.

[3] Alvarez, F., D. Argente and F. Lippi. 2020. "A Simple Planning Problem for COVID19 Lockdown." University of Chicago mimeo.

[4] Aruoba, S.B., M.A. Davis and R. Wright. 2016. "Homework in Monetary Economics: Inflation, Home Production, and the Production of Homes." Review of Economic Dynamics, 21: 105-124.

[5] Becker, G.S. 1965. "A Theory of the Allocation of Time." Economic Journal, 75: 493-517.

[6] Benhabib, J., R. Rogerson and R. Wright. 1991. "Homework in Macroeconomics: Household Production and Aggregate Fluctuations." Journal of Political Economy, 85: 1099-1123.

[7] Berger, D., K. Herkenhoff and S. Mongey. 2020. "An SEIR Infectious Disease Model with Testing and Conditional Quarantine." Duke University mimeo.

[8] Bethune, Z. and A. Korinek. 2020. "COVID-19 Infection Externalities: Trading Off Lives vs. Livelihoods." University of Virginia manuscript.

[9] Cochrane, J. C. 2020. "An SIR Model with Behavior." https://johnhcochrane.blogspot.com/2020/05/an-sir-model-with-behavior.html.

[10] Davis J.T., M. Chinazzi, N. Perra, K. Mu, A. Pastore y Piontti, M. Ajelli, N. E. Dean, C. Gioannini, M. Litvinova, S. Merler, L. Rossi, K. Sun, X. Xiong, M. E. Halloran, I. M Longini Jr., C. Viboud and A. Vespignani. 2020. "Estimating the Establishment of Local Transmission and the Cryptic Phase of the COVID-19 Pandemic in the USA." medRxiv 2020.07.06.20140285.

[11] Eichenbaum, M.S., S. Rebelo and M. Trabandt. 2020a. "The Macroeconomics of Epidemics." NBER Working Paper No. 26882. 
[12] Eichenbaum, M.S., S. Rebelo and M. Trabandt. 2020b. "The Macroeconomics of Testing and Quarantining." Northwestern University manuscript.

[13] Eichenbaum, M.S., S. Rebelo and M. Trabandt. 2020c. "Epidemics in the Neoclassical and New-Keynesian Models." Northwestern University manuscript.

[14] Farhi, E. and X. Gabaix. 2020. "Optimal Taxation with Behavioral Agents." American Economic Review, 110: 298-336.

[15] Ferguson, N.M., D.A.T. Cummings, C. Fraser, J.C. Cajka, P.C. Cooley and D.S. Burke. 2006. "Strategies for Mitigating an Influenza Pandemic." Nature, 442: 448452.

[16] Gabaix, X. 2014. "A Sparsity-Based Model of Bounded Rationality." Quarterly Journal of Economics, 129: 1661-1710.

[17] Gabaix, X. 2016. "Behavioral Macroeconomics via Sparse Dynamic Programming." NBER Working Paper No. 21848.

[18] Gabaix, X. 2019. "Behavioral Inattention." Handbook of Behavioral Economics, 2: 261-344.

[19] Gabaix, X. 2020. "A Behavioral New Keynesian Model." American Economic Review, 110: 2271-2327.

[20] Gans, J. 2020. "The Economic Consequences of $\mathrm{R}=1$ : Towards a Workable Behavioural Epidemiological Model of Pandemics." University of Torotno mimeo.

[21] Gayle, G.L. and A. Shephard. 2019. "Optimal Taxation, Marriage, Home Production, and Family Labor Supply." Econometrica, 87: 291-326.

[22] Glover, A., J. Heathcote, D. Krueger and J.V. Rios-Rull. 2020. "Health versus Wealth: On the Distribution Effects of Controlling a Pandemic." University of Pennsylvania mimeo.

[23] Guerrieri, V., G. Lorenzoni, L. Straub and I. Werning. 2020. "Macroeconomic Implications of COVID-19: Can Negative Supply Shocks Cause Demand Shortages?" Northwestern University mimeo.

[24] Greenwood J. and Z. Hercovitz. 1991. "The Allocation of Capital and Time Over the Business Cycle." Journal of Political Economy, 99: 1188-1214. 
[25] Gronau, R. 1977. "Leisure, Home Production, and Work-the Theory of the Allocation of Time Revisited." Journal of Political Economy, 99: 1166-1187.

[26] Gronau, R. 1986. "Home Production - A Survey." Handbook of Labor Economics, 1: $273-304$.

[27] Gruber, J. and B. Koszegi. 2001. "Is Addiction "Rational"? Theory and Evidence." Quarterly Journal of Economics, 116: 1261-1303.

[28] Hall, R.E. and C.I. Jones. 2007. "The Value of Life and the Rise in Health Spending." Quarterly Journal of Economics, 122: 39-72.

[29] Hall, R.E, C. Jones and P. Klenow. 2020. "Trading Off Consumption and COVID-19 Deaths." Stanford University manuscript.

[30] Halpern, S.D., R.D. Truog and F.G. Miller. 2020. "Cognitive Bias and Public Health Policy During the COVID-19 Pandemic." Journal of the American Medical Association, 324: 337-338.

[31] Ing, A.J., C. Cocks and J.P. Green. 2020. "COVID-19: In the Footsteps of Ernest Shackleton." Thorax, 75: 693-694.

[32] Jones, C. J., T. Philippon and V. Venkateswaran. 2020. "Optimal Mitigation Policies in a Pandemic: Social Distancing and Working from Home." NBER Working Paper. No. 26984.

[33] Kanal, D. and J.S. Kornegay. 2019. "Accounting for Household Production in the National Accounts. An Update, 1965-2017." Survey of Current Business, 99(6).

[34] Kaplan, G., B. Moll and G. Violante. 2020. "Pandemics According to HANK." University of Chicago mimeo.

[35] Kermack, W.O. and A.G. McKendrick. 1927. "A Contribution to the Mathematical Theory of Epidemics." Proceedings of the Royal Society of London, series A, 115 (772): 700-721.

[36] Krueger, D., H. Uhlig and T. Xie. 2020. "Macroeconomic Dynamics and Reallocation in an Epidemic." University of Chicago manuscript. 
[37] Li. R., S. Pei, B. Chen, Y. Song, T. Zhang, W. Yang and J. Shaman. 2020. "Substantial Undocumented Infection Facilitates the Rapid Dissemination of Novel Coronavirus (SARS-CoV-2)." Science, 268: 489-493.

[38] Miller, J.C. 2020. "A Note on the Derivation of Epidemic Final Sizes." Bulletin of Mathematical Biology, 74: 2125-2141.

[39] Moghadas, S.M., M.C. Fitzpatrick, P. Sah, A. Pandey, A. Shoukat, B.H. Singer and A.P. Galvani. 2020. "The Implications of Silent Transmission for the Control of COVID-19 Outbreaks." Proceedings of the National Academy of Sciences, 117: 17513-17515.

[40] Poletti, P., M. Tirani, D. Cereda, F. Trentini, G. Guzzetta, G. Sabatino, V. Marziano, A. Castrofino, F. Grosso, G. Del Castillo, R. Piccarreta, ATS Lombardy COVID-19 Task Force, A. Andreassi, A. Melegaro, M. Gramegna, M. Ajelli and S. Merler. 2020. "Probability of Symptoms and Critical Disease after SARS-CoV-2 Infection." arXiv:2006.08471.

[41] Romer, P. 2020. "Road to Responsibly Reopen America.," New York University Mimeo.

[42] Toxvaerd, F. 2020. "Equilibrium Social Distancing." Cambridge University mimeo.

[43] Tversky, A. and D. Kahneman. 1974. "Judgment under Uncertainty: Heuristics and Biases." Science, 185: 1124-1130. 


\section{Appendix}

\section{A Solving the pre-infection economy model}

Note that since there are no intertemporal decisions in this economy, we can solve the model period by period. Specifically, the first-order conditions for the representativeagents' problem in the pre-infection economy are:

$$
\begin{gathered}
\omega_{h}^{c} c_{t}^{-1}\left(\frac{c_{t}}{c_{h, t}}\right)^{1 / \eta}-\lambda_{b h, t}=0, \\
c_{t}^{-1}\left(\frac{c_{t}}{c_{k, t}}\right)^{1 / \eta}-\lambda_{b k, t}=0, \\
\theta_{l} \omega_{h}^{l} l_{t}^{-1}\left(\frac{l_{t}}{l_{h, t}}\right)^{1 / \sigma}+\lambda_{a, t}=0, \\
\theta_{l} l_{t}^{-1}\left(\frac{l_{t}}{l_{k, t}}\right)^{1 / \sigma}+\lambda_{a, t}=0, \\
\lambda_{a, t}+A_{h} \lambda_{b h, t}=0, \\
\lambda_{a, t}+A_{k} \lambda_{b k, t}=0,
\end{gathered}
$$

where $\lambda_{a, t}, \lambda_{b h, t}$, and $\lambda_{b h, t}$ are the Lagrange multipliers associated with constraints (2), (3) and (4), respectively.

Solving for $\lambda_{a, t}$ in equations (62) and (63) and rearranging, we find that:

$$
\lambda_{a, t}=-\theta_{l} \omega_{h}^{l} l_{t}^{-1}\left(\frac{l_{t}}{l_{h, t}}\right)^{1 / \sigma}=-\theta_{l} l_{t}^{-1}\left(\frac{l_{t}}{l_{k, t}}\right)^{1 / \sigma},
$$

and, consequently the ratio of home to social leisure satisfies:

$$
\frac{l_{h, t}}{l_{k, t}}=\left(\omega_{h}^{l}\right)^{\sigma}
$$

Substituting equation (66) into the expression for $l_{t}$ in equation (7) and dividing by $l_{k, t}$, we have

$$
\begin{aligned}
\frac{l_{t}}{l_{k, t}} & =\left[\omega_{h}^{l}\left(\frac{l_{h, t}}{l_{k, t}}\right)^{\frac{\sigma-1}{\sigma}}+1\right]^{\frac{\sigma}{\sigma-1}} \\
& =\left[\omega_{h}^{l}\left(\omega_{h}^{l}\right)^{\sigma-1}+1\right]^{\frac{\sigma}{\sigma-1}} \\
& =\left[\left(\omega_{h}^{l}\right)^{\sigma}+1\right]^{\frac{\sigma}{\sigma-1}}
\end{aligned}
$$

and substituting into (63), we get

$$
\lambda_{a, t}=-\theta_{l} l_{t}^{-1}\left(\frac{l_{t}}{l_{k, t}}\right)^{1 / \sigma}=-\theta_{l}\left[\left(\omega_{h}^{l}\right)^{\sigma}+1\right]^{-1} l_{k, t}^{-1} .
$$


From equations (64) and (65), we have that:

$$
\begin{gathered}
\lambda_{a, t}=-A_{h} \lambda_{b h, t}=-A_{k} \lambda_{b k, t} \\
A_{h} \omega_{h}^{c} c_{t}^{-1}\left(\frac{c_{t}}{c_{h, t}}\right)^{1 / \eta}=A_{k} c_{t}^{-1}\left(\frac{c_{t}}{c_{k, t}}\right)^{1 / \eta} \\
\frac{c_{h, t}}{c_{k, t}}=\left(\frac{A_{h} \omega_{h}^{c}}{A_{k}}\right)^{\eta}
\end{gathered}
$$

and substituting the expressions for the consumption of the home and market goods in the budget constraints in equations (3) and (4), we arrive at:

$$
\frac{n_{h, t}}{n_{k, t}}=\left(\frac{A_{h}}{A_{k}}\right)^{\eta-1}\left(\omega_{h}^{c}\right)^{\eta} .
$$

Similarly, substituting equation (68) into the expression for $c_{t}$ in equation (6) and dividing by $c_{k, t}$, we have

$$
\begin{aligned}
\frac{c_{t}}{c_{k, t}} & =\left[\omega_{h}^{c}\left(\frac{A_{h} \omega_{h}^{c}}{A_{k}}\right)^{\eta-1}+1\right]^{\frac{\eta}{\eta-1}}, \\
& =\left[\left(\frac{A_{h}}{A_{k}}\right)^{\eta-1}\left(\omega_{h}^{c}\right)^{\eta}+1\right]^{\frac{\eta}{\eta-1}} .
\end{aligned}
$$

substituting this expression and the budget constraint for the market good in equation (3) into (63) we get the Lagrange multiplier that is associated with the budget constraint for the market good:

$$
\lambda_{b k, t}=c_{t}^{-1}\left(\frac{c_{t}}{c_{k, t}}\right)^{1 / \eta}=\left[\left(\frac{A_{h}}{A_{k}}\right)^{\eta-1}\left(\omega_{h}^{c}\right)^{\eta}+1\right]^{-1} A_{k}^{-1} n_{k, t}^{-1} .
$$

Substituting the expressions for $\lambda_{a, t}$, the Lagrange multiplier for the time budget constraint, and $\lambda_{b k, t}$, the Lagrange multiplier associated with the budget constraint for the market good, into equation (65), we obtain that

$$
\begin{gathered}
\lambda_{a, t}=-A_{k} \lambda_{b k, t}, \\
\theta_{l}\left[\left(\omega_{h}^{l}\right)^{\sigma}+1\right]^{-1} l_{k, t}^{-1}=\left[\left(\frac{A_{h}}{A_{k}}\right)^{\eta-1}\left(\omega_{h}^{c}\right)^{\eta}+1\right]^{-1} n_{k, t}^{-1}, \\
l_{k, t}=\theta_{l}\left[\left(\omega_{h}^{l}\right)^{\sigma}+1\right]^{-1}\left[\left(\frac{A_{h}}{A_{k}}\right)^{\eta-1}\left(\omega_{h}^{c}\right)^{\eta}+1\right] n_{k, t} .
\end{gathered}
$$

Finally, substituting equations (66), (69) and (71) into the time budget constraint in equation (2), we can solve for the amount of time the representative agent works in the 
market sector

$$
n_{k . t}=\frac{1}{\left(1+\theta_{l}\right)} \frac{1}{\left[\left(\frac{A_{h}}{A_{k}}\right)^{\eta-1}\left(\omega_{h}^{c}\right)^{\eta}+1\right]} \bar{n},
$$

which is equation (8) in the main text. The rest of the representative agent's time allocation in equations $(9),(10),(11)$ can be obtained by substituting equation (72) into (66), (69) and (71), respectively.

\section{B Derivatives of subjective probabilities}

We have that the subjective probability of being infected with severe symptoms at time $t+1$, conditional on being an individual with an unknown health status, and its derivative with respect to $\widetilde{\tau}_{t}$ are given by

$$
\begin{aligned}
\widetilde{p}\left(i_{t+1} \mid v_{t}\right) & =\tilde{\pi}_{i} \widetilde{\tau}_{t} \widetilde{p}\left(s_{t} \mid v_{t}\right), \\
\frac{\partial \widetilde{p}\left(i_{t+1} \mid v_{t}\right)}{\partial \widetilde{\tau}_{t}} & =\widetilde{\pi}_{i} \widetilde{p}\left(s_{t} \mid v_{t}\right) .
\end{aligned}
$$

The subjective probability of being mildly symptomatic and isolated at time $t+1$ and its derivative are

$$
\begin{aligned}
\widetilde{p}\left(q_{t+1} \mid v_{t}\right) & =\left(1-\widetilde{\pi}_{i}\right) \alpha \widetilde{\tau} t \tilde{p}\left(s_{t} \mid v_{t}\right), \\
\frac{\partial \widetilde{p}\left(q_{t+1} \mid v_{t}\right)}{\partial \widetilde{\tau}_{t}} & =\left(1-\pi_{i}\right) \alpha \widetilde{p}\left(s_{t} \mid v_{t}\right) .
\end{aligned}
$$

Similarly, we have that the subjective probability of remaining in the unknown health status category at time $t+1$ (and its derivative) are

$$
\begin{aligned}
\widetilde{p}\left(v_{t+1} \mid v_{t}\right) & =1-\widetilde{p}\left(i_{t+1} \mid v_{t}\right)-\widetilde{p}\left(q_{t+1} \mid v_{t}\right), \\
\frac{\partial \widetilde{p}\left(v_{t+1} \mid v_{t}\right)}{\partial \widetilde{\tau}_{t}} & =-\frac{\partial \widetilde{p}\left(i_{t+1} \mid v_{t}\right)}{\partial \widetilde{\tau}_{t}}-\frac{\partial \widetilde{p}\left(q_{t+1} \mid v_{t}\right)}{\partial \widetilde{\tau}_{t}} \\
& =-\left[\widetilde{\pi}_{i}+\left(1-\widetilde{\pi}_{i}\right) \alpha\right] \widetilde{p}\left(s_{t} \mid v_{t}\right) .
\end{aligned}
$$

Further, we have that the subjective probability of being infected with severe symptoms at time $t+2$, conditional on being an individual with an unknown health status at time $t$, and its derivative with respect to $\widetilde{\tau}_{t}$ are

$$
\begin{aligned}
\widetilde{p}\left(i_{t+2} \mid v_{t}\right) & =\widetilde{\pi}_{i} \widetilde{\tau}_{t+1}\left(1-\widetilde{\tau}_{t}\right) \widetilde{p}\left(s_{t} \mid v_{t}\right)+\left(1-\widetilde{\pi}_{r}^{i}-\widetilde{\pi}_{d}^{i}\right) \widetilde{\pi}_{i} \widetilde{\tau}_{t} \widetilde{p}\left(s_{t} \mid v_{t}\right), \\
& =\widetilde{\pi}_{i} \widetilde{\tau}_{t+1} \widetilde{p}\left(s_{t} \mid v_{t}\right)+\left[\left(1-\widetilde{\pi}_{r}^{i}-\widetilde{\pi}_{d}^{i}\right)-\widetilde{\tau}_{t+1}\right] \widetilde{\pi}_{i} \widetilde{\tau}_{t} \widetilde{p}\left(s_{t} \mid v_{t}\right), \\
\frac{\partial \widetilde{p}\left(i_{t+2} \mid v_{t}\right)}{\partial \widetilde{\tau}_{t}} & =\left[\left(1-\widetilde{\pi}_{r}^{i}-\widetilde{\pi}_{d}^{i}\right)-\widetilde{\tau}_{t+1}\right] \widetilde{\pi}_{i} \widetilde{p}\left(s_{t} \mid v_{t}\right), \\
& =\left[\left(1-\widetilde{\pi}_{r}^{i}-\widetilde{\pi}_{d}^{i}\right)-\widetilde{\tau}_{t+1}\right] \frac{\partial \widetilde{p}\left(i_{t+1} \mid v_{t}\right)}{\partial \widetilde{\tau}_{t}}
\end{aligned}
$$


The subjective probability of being mildly symptomatic and isolated at time $t+2$, conditional on being an individual with an unknown health status at time $t$, and its derivative are given by

$$
\begin{aligned}
\widetilde{p}\left(q_{t+2} \mid v_{t}\right) & =\left(1-\widetilde{\pi}_{i}\right) \alpha \widetilde{\tau}_{t+1}\left(1-\widetilde{\tau}_{t}\right) \widetilde{p}\left(s_{t} \mid v_{t}\right)+\left(1-\widetilde{\pi}_{r}^{a}\right)\left(1-\widetilde{\pi}_{i}\right) \alpha \widetilde{\tau}_{t} \tilde{p}\left(s_{t} \mid v_{t}\right) . \\
& =\left(1-\widetilde{\pi}_{i}\right) \alpha \widetilde{\tau}_{t+1} \widetilde{p}\left(s_{t} \mid v_{t}\right)+\left[\left(1-\widetilde{\pi}_{r}^{a}\right)-\widetilde{\tau}_{t+1}\right]\left(1-\widetilde{\pi}_{i}\right) \alpha \widetilde{\tau}_{t} \widetilde{p}\left(s_{t} \mid v_{t}\right), \\
\frac{\partial p\left(q_{t+2} \mid u_{t}\right)}{\partial \widetilde{\tau}_{t}} & =\left[\left(1-\widetilde{\pi}_{r}^{a}\right)-\widetilde{\tau}_{t+1}\right]\left(1-\widetilde{\pi}_{i}\right) \alpha \widetilde{p}\left(s_{t} \mid v_{t}\right), \\
& =\left[\left(1-\widetilde{\pi}_{r}^{a}\right)-\widetilde{\tau}_{t+1}\right] \frac{\partial \widetilde{p}\left(q_{t+1} \mid v_{t}\right)}{\partial \widetilde{\tau}_{t}} .
\end{aligned}
$$

The subjective probability of dying at time $t+2$, and its derivative, satisfy

$$
\begin{aligned}
\widetilde{p}\left(d_{t+2} \mid v_{t}\right) & =\widetilde{\pi}_{r}^{d} \widetilde{\pi}_{i} \widetilde{\tau}_{t} \widetilde{p}\left(s_{t} \mid v_{t}\right) \\
\frac{\partial \widetilde{p}\left(d_{t+2} \mid v_{t}\right)}{\partial \widetilde{\tau}_{t}} & =\widetilde{\pi}_{r}^{d} \widetilde{\pi}_{i} \widetilde{p}\left(s_{t} \mid v_{t}\right) \\
& =\widetilde{\pi}_{r}^{d} \frac{\partial \widetilde{p}\left(i_{t+1} \mid v_{t}\right)}{\partial \widetilde{\tau}_{t}} .
\end{aligned}
$$

The subjective probability of being recovered and detected at time $t+2$, and its derivative, satisfy

$$
\begin{aligned}
\widetilde{p}\left(r_{t+2} \mid v_{t}\right) & =\widetilde{\pi}_{r}^{i} \widetilde{\pi}_{i} \widetilde{\tau}_{t} \widetilde{p}\left(s_{t} \mid v_{t}\right)+\widetilde{\pi}_{r}^{a}\left(1-\widetilde{\pi}_{i}\right) \alpha \widetilde{\tau} t \tilde{p}\left(s_{t} \mid v_{t}\right) \\
\frac{\partial \widetilde{p}\left(r_{t+2} \mid v_{t}\right)}{\partial \widetilde{\tau}_{t}} & =\widetilde{\pi}_{r}^{i} \widetilde{\pi}_{i} \widetilde{p}\left(s_{t} \mid v_{t}\right)+\widetilde{\pi}_{r}^{a}\left(1-\widetilde{\pi}_{i}\right) \alpha \widetilde{p}\left(s_{t} \mid v_{t}\right) \\
& =\widetilde{\pi}_{r}^{i} \frac{\partial \widetilde{p}\left(i_{t+1} \mid v_{t}\right)}{\partial \widetilde{\tau}_{t}}+\widetilde{\pi}_{r}^{a} \frac{\partial \widetilde{p}\left(q_{t+1} \mid v_{t}\right)}{\partial \widetilde{\tau}_{t}}
\end{aligned}
$$

Finally, we have that the subjective probability of remaining in the unknown health status category at time $t+2$, and its derivative, are given by:

$$
\begin{aligned}
\widetilde{p}\left(v_{t+2} \mid v_{t}\right) & =1-\widetilde{p}\left(i_{t+2} \mid v_{t}\right)-\widetilde{p}\left(q_{t+2} \mid v_{t}\right)-\widetilde{p}\left(r_{t+2} \mid v_{t}\right)-\widetilde{p}\left(d_{t+2} \mid v_{t}\right) \\
\frac{\partial \widetilde{p}\left(v_{t+2} \mid v_{t}\right)}{\partial \widetilde{\tau}_{t}} & =-\frac{\partial \widetilde{p}\left(i_{t+2} \mid v_{t}\right)}{\partial \widetilde{\tau}_{t}}-\frac{\partial p\left(q_{t+2} \mid u_{t}\right)}{\partial \widetilde{\tau}_{t}}-\frac{\partial \widetilde{p}\left(d_{t+2} \mid v_{t}\right)}{\partial \widetilde{\tau}_{t}}-\frac{\partial \widetilde{p}\left(r_{t+2} \mid v_{t}\right)}{\partial \widetilde{\tau}_{t}} .
\end{aligned}
$$


Table 1

Parameter values

\begin{tabular}{ccl}
\hline Parameter & Value & Description \\
Pre-epidemic & parameters & \\
$\theta_{0}$ & -6.136 & Momentary utility function constant \\
$\theta_{l}$ & $2 / 3$ & Labour supply parameter \\
$\eta$ & 3 & Elasticity of consumption substitution \\
$\omega_{h}^{c}$ & 1.260 & Home good preference parameter \\
$\sigma$ & 3 & Elasticity of leisure substitution \\
$\omega_{h}^{l}$ & 1.442 & Home leisure preference parameter \\
$A_{k}$ & $2.789 \times 10^{3}$ & Productivity market good \\
$A_{h}$ & $1.394 \times 10^{3}$ & Productivity home good \\
$\bar{n}$ & 1 & Time available for work and leisure \\
& & \\
Epidemic & parameters & \\
$\pi_{i}$ & 0.25 & Probability of developing severe symptoms \\
$\pi_{r}^{i}$ & 0.496 & Recovery rate conditional on severe symptoms \\
$\pi_{d}^{i}$ & 0.004 & Mortality rate conditional on severe symptoms \\
$\pi_{r}^{a}$ & 0.500 & Recovery rate conditional on mild symptoms \\
$\pi_{n}$ & 2.604 & Market work infection intensity \\
$\pi_{l}$ & 41.667 & Social leisure infection intensity \\
$\pi_{h}$ & 3.333 & Home infection intensity \\
$\bar{n}^{i}$ & $2 / 3$ & Time available if infected with severe form of the disease \\
$\varepsilon$ & $10^{-4}$ & Initial size of the outbreak \\
Behavioral parameters & \\
$\bar{m}$ & $1 / 2$ & Future inattention parameter \\
\hline & & \\
& & \\
& &
\end{tabular}


Figure 1. Natural history of the disease

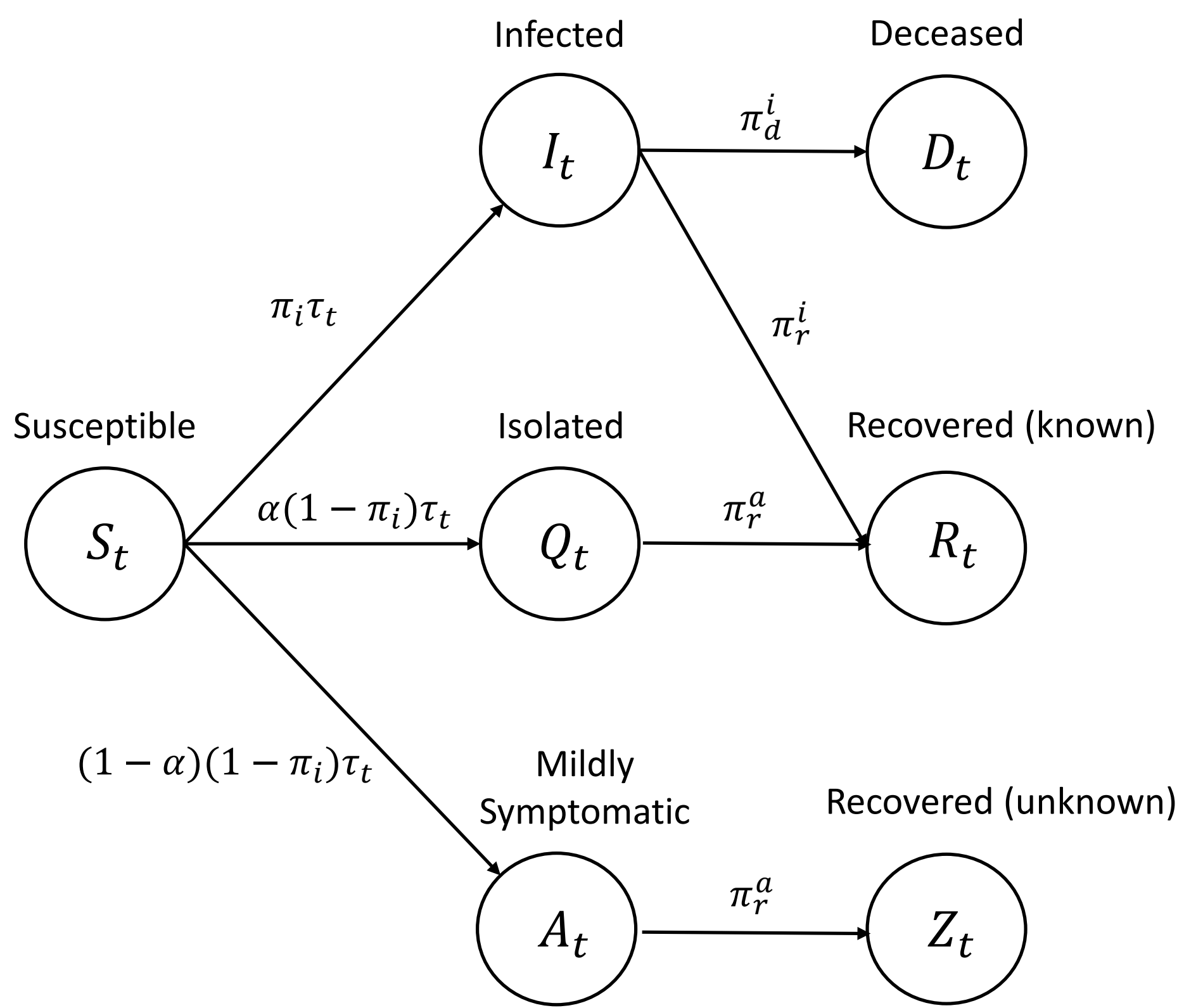


Figure 2A. Health Dynamics - Model with Naive Agents
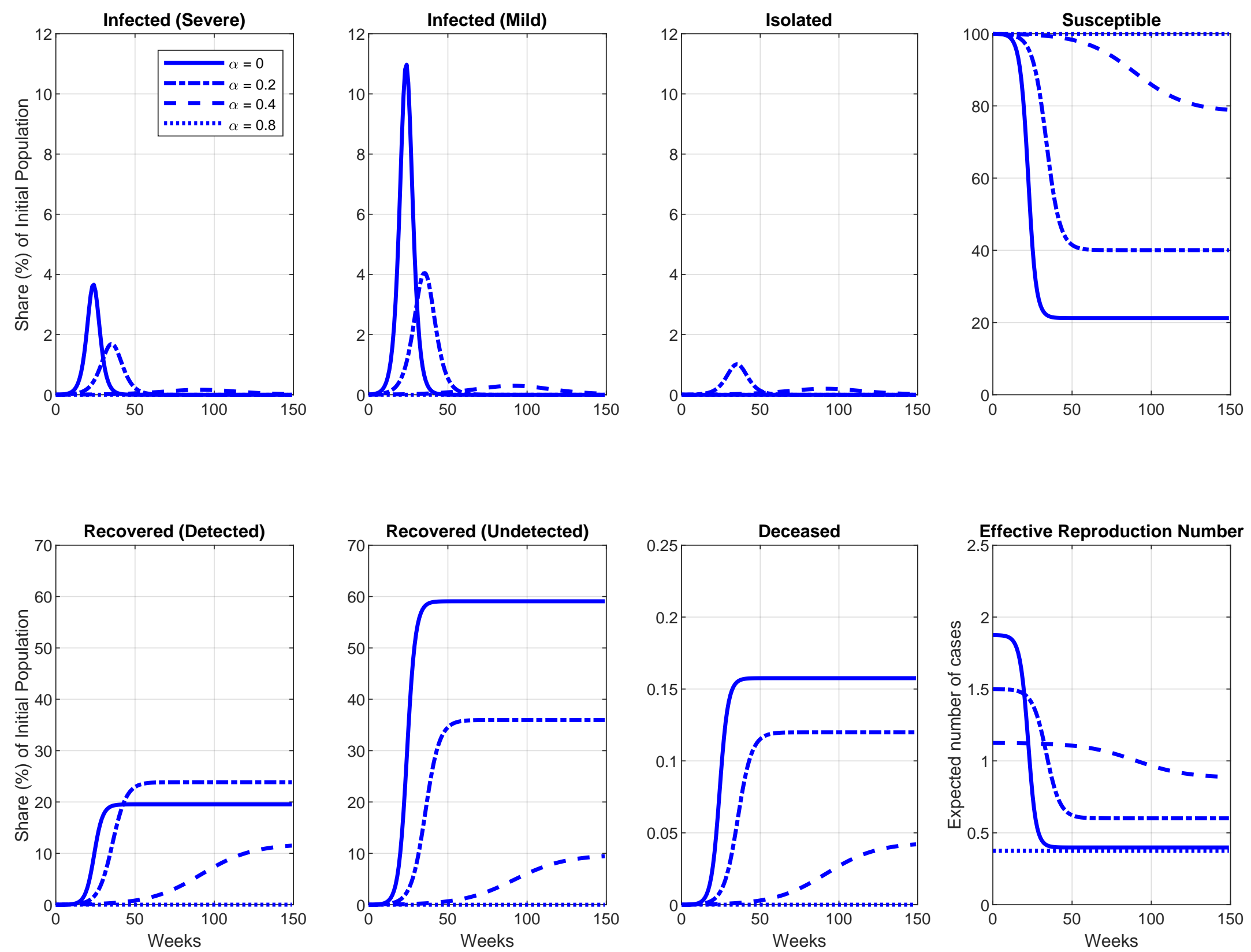
Figure 2B. Time Allocation for a Naive Agent with Unknown Health Status
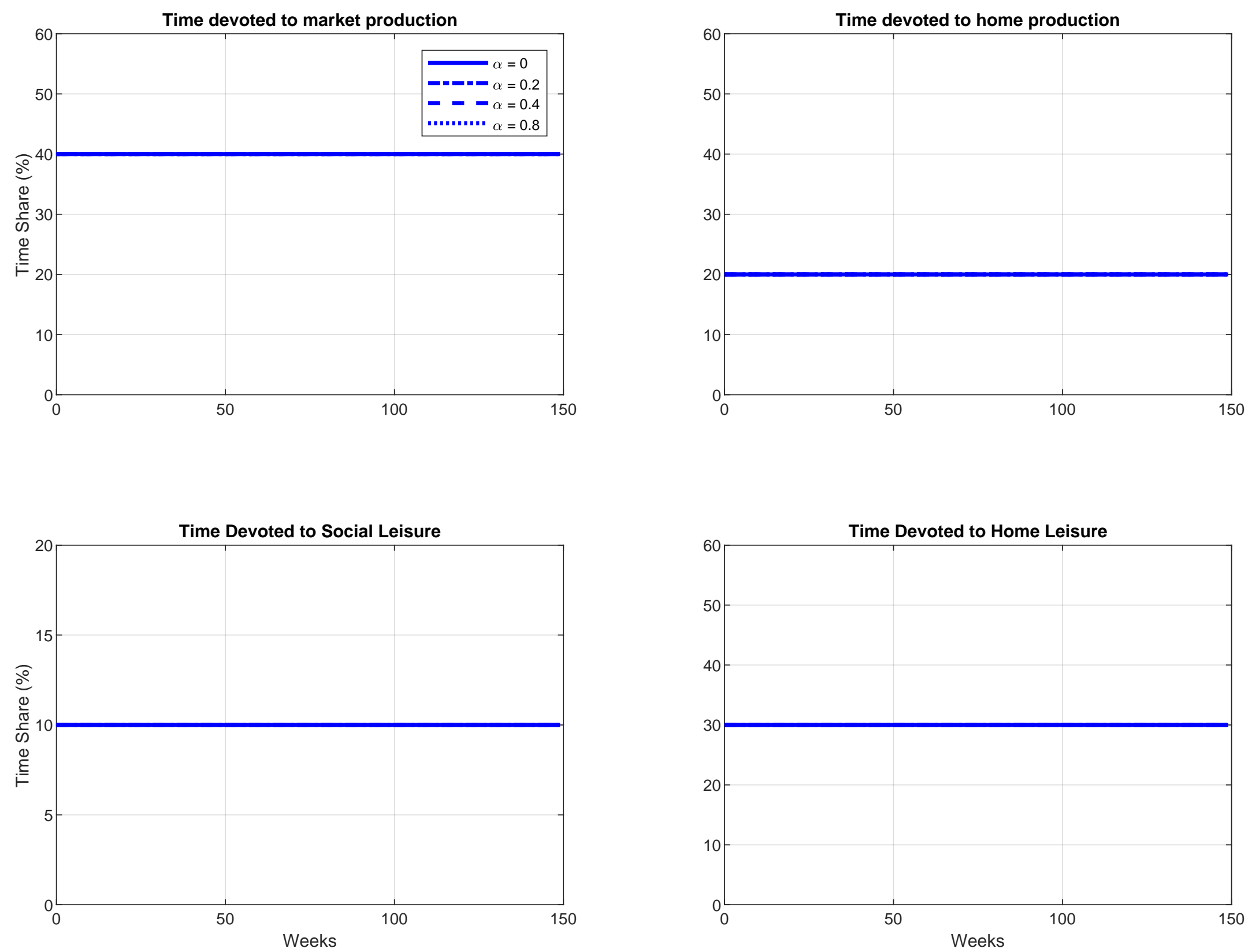
Figure 2C. Consumption Dynamics - Model with Naive Agents
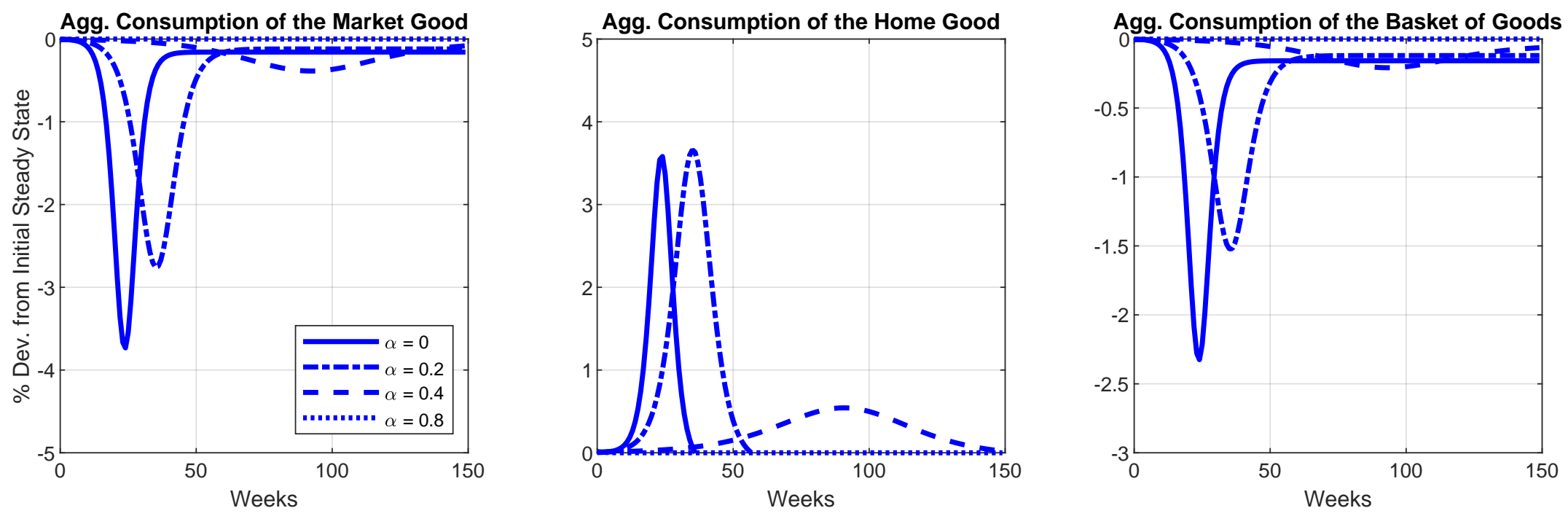
Figure 3A. Health Dynamics - Model with Rational Agents
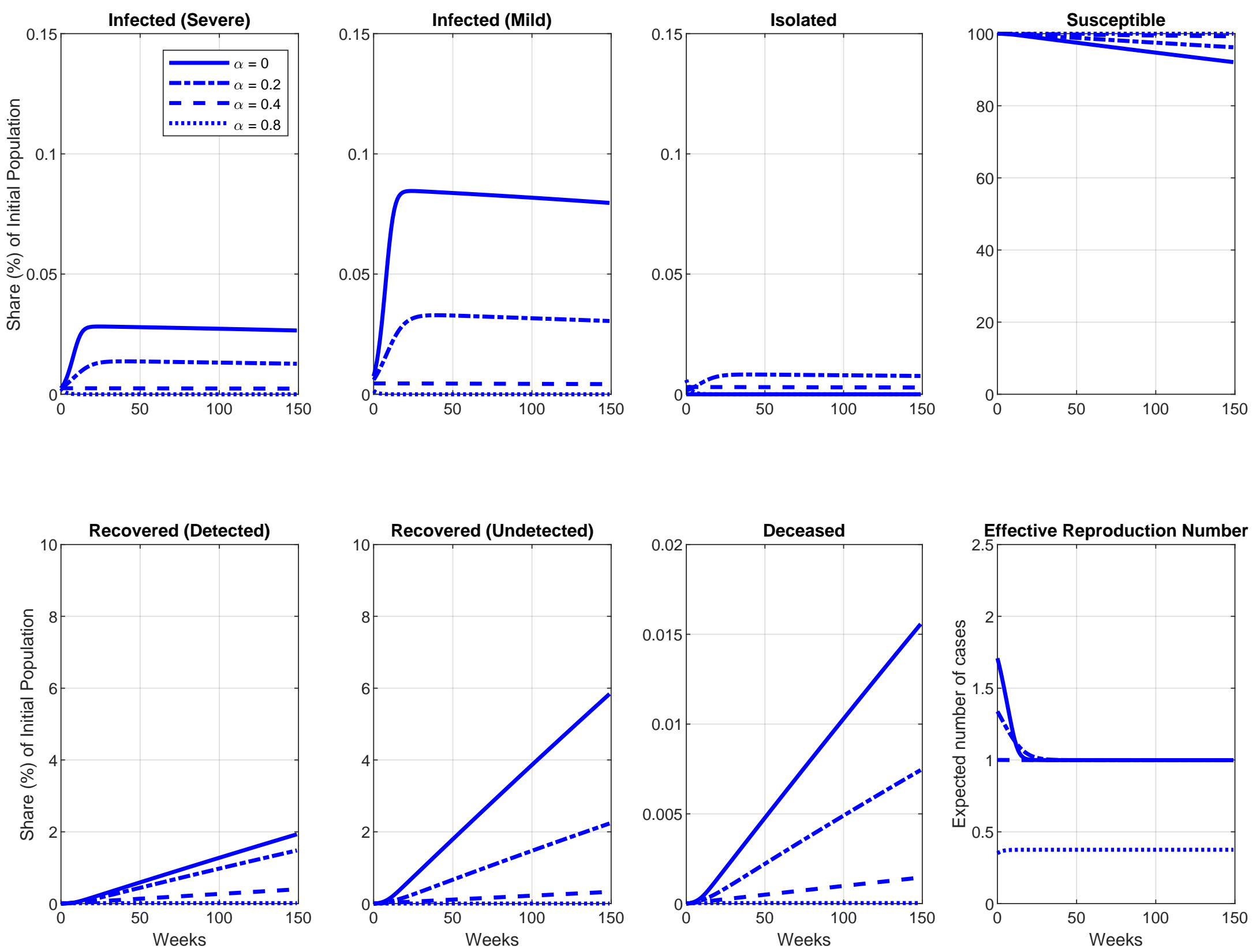
Figure 3B. Time Allocation for a Rational Agent with Unknown Health Status
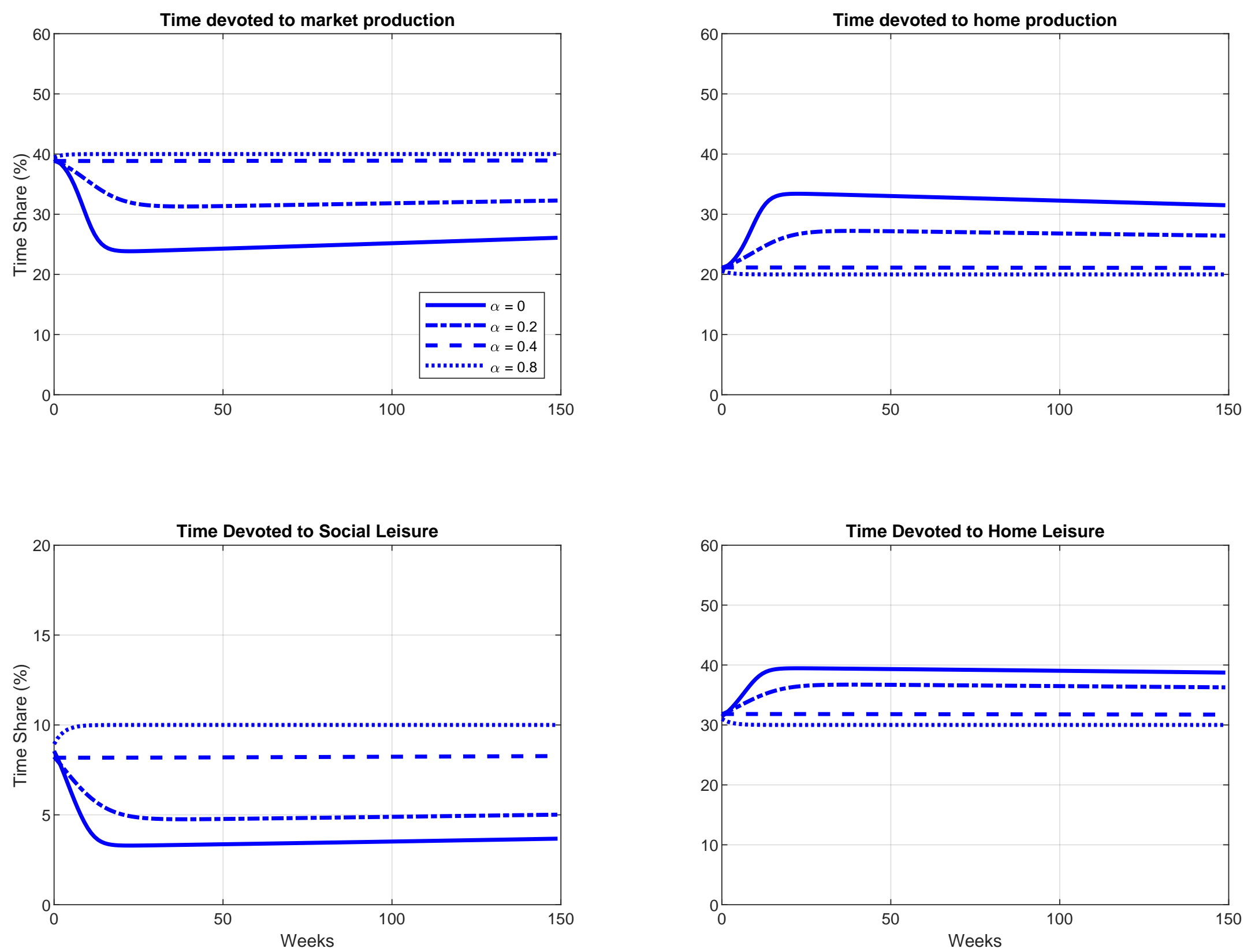
Figure 3C. Consumption Dynamics - Model with Rational Agents
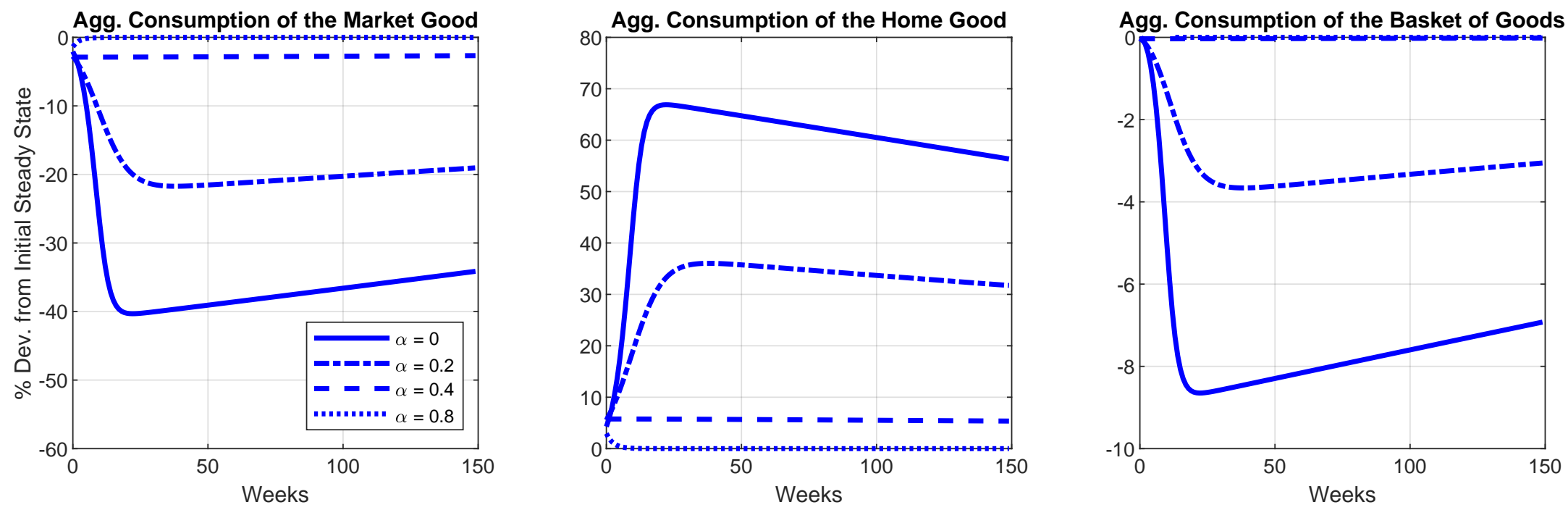
Figure 4A. Health Dynamics - Model with Optimistic Agents
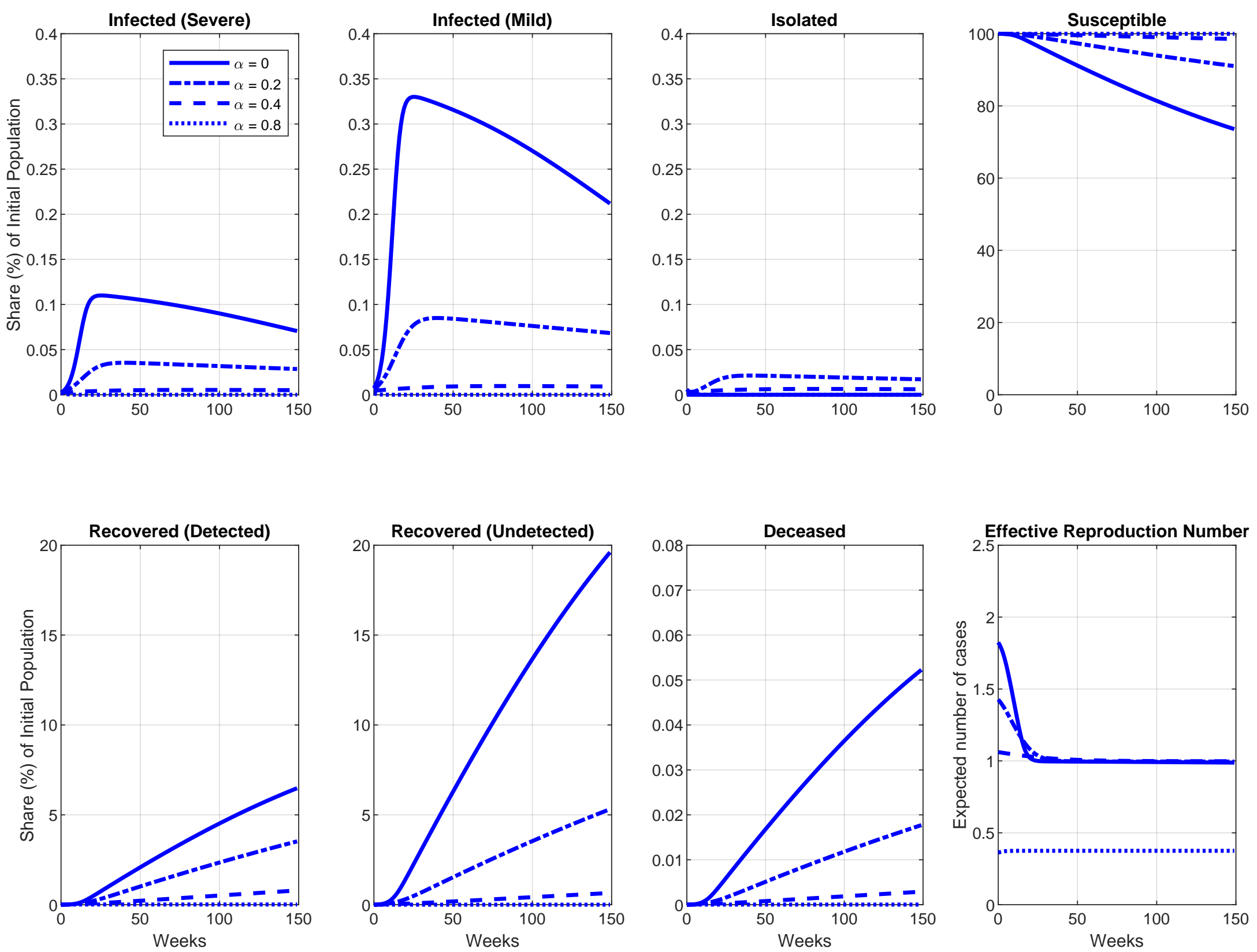
Figure 4B. Time Allocation for an Optimistic Agent with Unknown Health Status
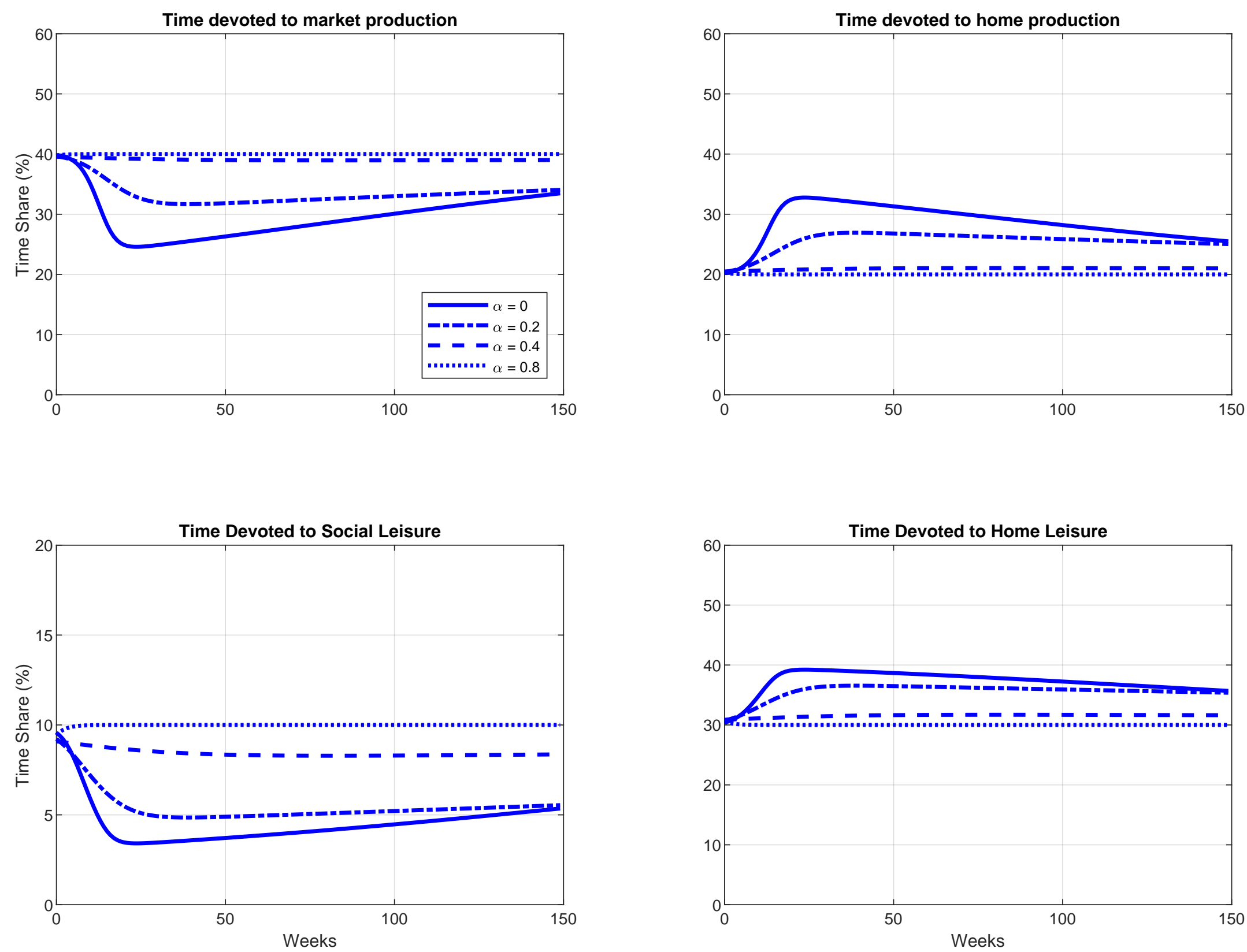
Figure 4C. Consumption Dynamics - Model with Optimistic Agents
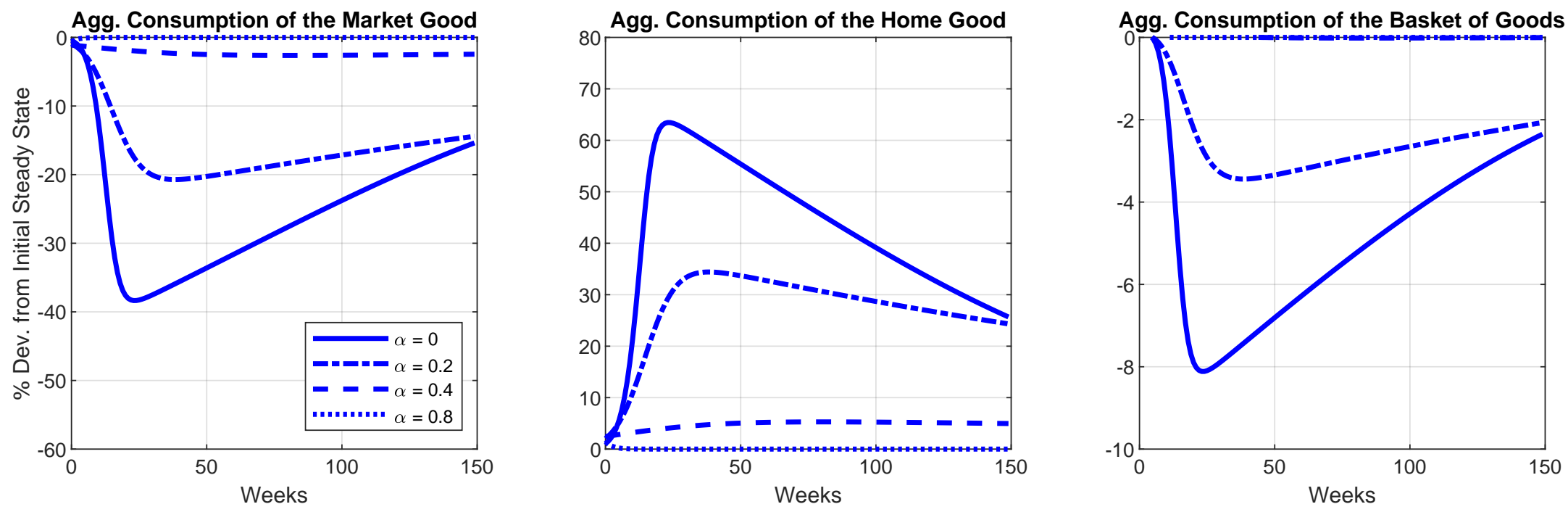
Figure 5A. Health Dynamics - Model with Time-Varying Inattentive Agents
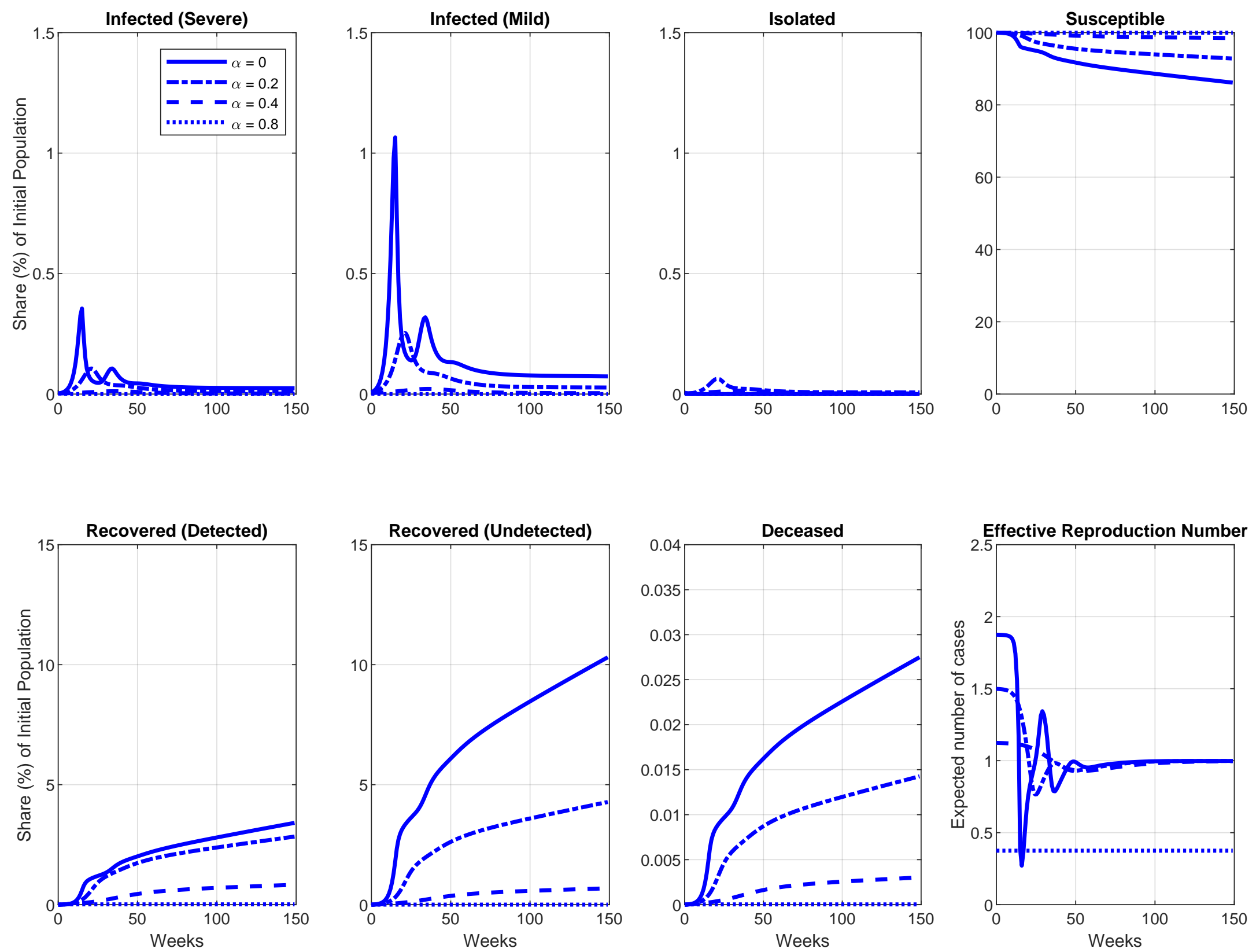
Figure 5B. Time Allocation for an Agent with Unknown Health Status and Time-Varying Inattention
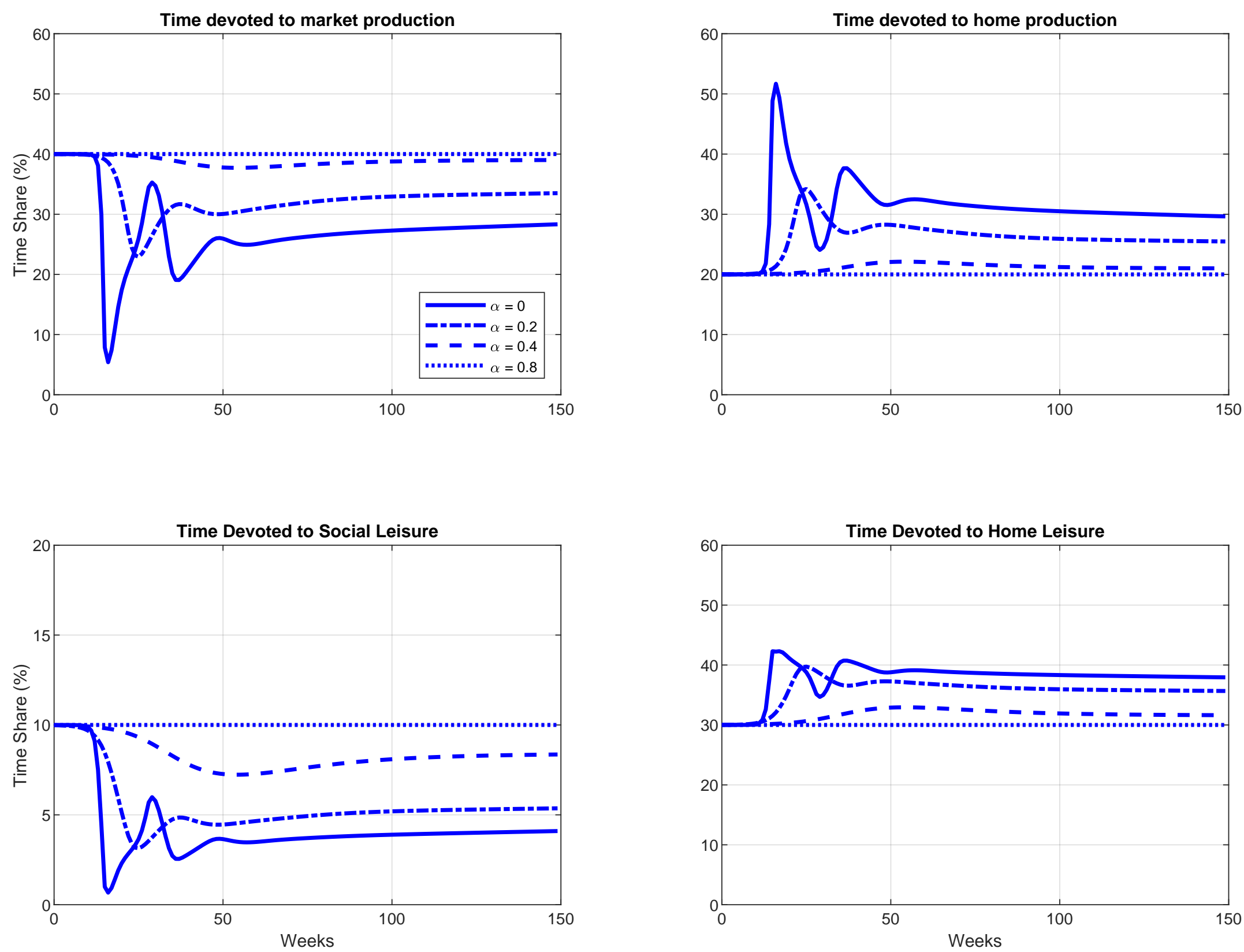
Figure 5C. Consumption Dynamics - Model with Time-Varying Inattentive Agents
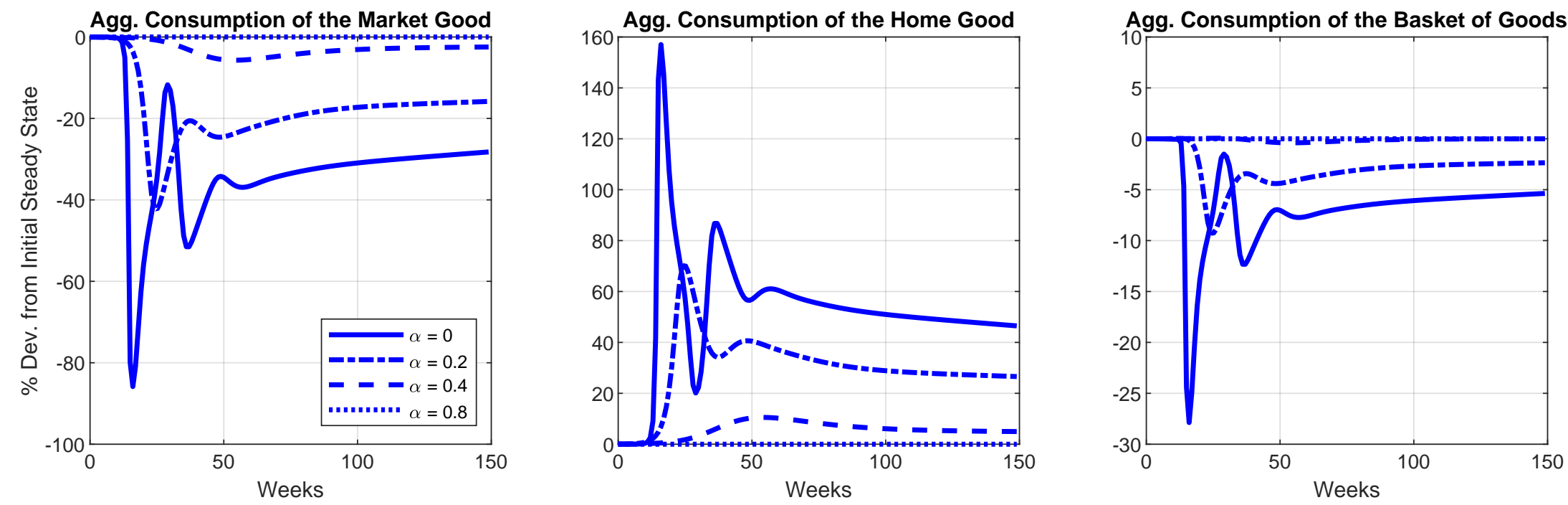